\title{
Joint optimisation of privacy and cost of in-app mobile user profiling and targeted ads
}

\author{
Imdad Ullah, Member, IEEE
}

\begin{abstract}
Online mobile advertising ecosystems provide advertising and analytics services that collect, aggregate, process and trade rich amount of consumer's personal data and carries out interests-based ads targeting, which raised serious privacy risks and growing trends of users feeling uncomfortable while using internet services. In this paper, we address user's privacy concerns by developing an optimal dynamic optimisation cost-effective framework for preserving user privacy for profiling, ads-based inferencing, temporal apps usage behavioral patterns and interest-based ads targeting. A major challenge in solving this dynamic model is the lack of knowledge of time-varying updates during profiling process. We formulate a mixed-integer optimisation problem and develop an equivalent problem to show that proposed algorithm does not require knowledge of time-varying updates in user behavior. Following, we develop an online control algorithm to solve equivalent problem using Lyapunov optimisation and to overcome difficulty of solving nonlinear programming by decomposing it into various cases and achieve trade-off between user privacy, cost and targeted ads. We carry out extensive experimentations and demonstrate proposed framework's applicability by implementing its critical components using POC 'System App'. We compare proposed framework with other privacy protecting approaches and investigate that it achieves better privacy and functionality for various performance parameters.
\end{abstract}

Index Terms—Privacy, Advertising, Optimisation, Privacy aware targeted mobile advertising services, Data privacy, Cost saving

\section{INTRODUCTION}

The online advertising companies enable user tracking with the help of millions of mobile applications (apps) offered via various app markets, profile users and enable targeted advertising where user's personal information plays an important role. The advertising ecosystems connect billions of mobile devices, including smartphones, tablets, and computer tablets, with the help of various tracking technologies to collect, process and map, and dissimilate user's private sensitive information. The strict laws imposed by the governments, in addition to abundance of anti-tracking tools/policies, the advertising companies are gradually obsoleting the use of third-party cookies used for interestbased targeting. Google announcement on Chrome's 'Cookie Apocalypse' to phase out support for third-party cookies by $2022^{1}$, similarly, Apple ${ }^{2}$ announced to update their tracking by introducing IDFA (Identification for Advertisers) opt-in overhaul, which will have significant impact over mobile ads targeting and in-app data attribution. Hence, instead of relying on third-party data, the advertisers and publishers tend towards first-party data, which has its own associated privacy concerns and will limit targeting vast majority of consumers. The advertisers and publishers are shifting towards maintaining data management platforms (DMPs) and demand-side platforms (DSPs) to brand their own data and measure performance in a cookie-less world. In addition,

- I. Ullah is with the College of Computer Engineering and Sciences, Prince Sattam bin Abdulaziz University, Al-Kharj 11942, Saudi Arabia. E-mail: i.ullah@psau.edu.sa

1. https://www.adviso.ca/en/blog/tech-en/cookie-apocalypse/

2. https://junction.cj.com/article/button-weighs-in-what-does-app les-idfa-opt-in-overhaul-mean-for-affiliate users are more concerned about their data in the long term e.g. Data they leave after death (Dad).

An important factor in online targeted advertising is to deliver relevant ads to users to achieve better view/click through rates without exposing their private and sensitive information, within or outside the advertising ecosystem. In its current working mechanism, the user tracking and advertising have raised significant privacy concerns, as suggested in a number of studies [1], [2], [3], [4], [5], [6], [7]. Other works show the extent to which consumer's activities are tracked by third-parties and across multiple apps [8], mobile devices leaking Personally Identifiable Information (PII) [9], [10], accessing user's sensitive information via APIs [11], and profile inference attacks based on monitoring ads [12]. Research studies indicate, unless consumers have specifically consented to it, that the ad/tracking companies evaluate user behavior and tailor advertising to reach specific audiences. The American self-regulatory authority, AdChoices ${ }^{3}$ program, presents a platform for consumers to opt-out of targeted ads and tracking done by the participating companies in WebChoices, however, this would result in less revenue, in addition to, presenting less relevant ads and lower click through rates, as evident in [13].

In this paper, we mainly focus on protecting user's privacy in mobile advertising ecosystem from profiling of specific private attributes that is potentially used by analytics companies for tracking user's behavior, the ads targeting based on these attributes, and user's apps usage behavior. The proposed framework provides an optimal privacy preserving profile management that is, in addition to providing above user privacy, cost effective and achieves an optimal trade-off between user privacy and targeted ads. In particular,

3. https: / /optout.aboutads.info/?c=2\&lang=EN 
the private attributes during profiling process dominate user profile, which can be lowered down to reduce its dominating factor, to produce disturbance in user profile, to control interest-based targeting, and to control temporal change in activity-based user profiling. Similarly, the use of newly suggested apps can be used to flatten the usage pattern of individuals to protect temporal user's apps usage behavior, which would be of particular scenario when users use mobile phone during business hours. Ideally, these apps can be run during when there is no activity in order to achieve an average usage of apps to actual usage patterns. However, a major challenge is to evaluate temporal change in user profiles and apps usage i.e. respectively the lack of knowledge of time-varying updates in user profiles, which we classy as updates caused by browsing history/searches, interactions with ads, and the types of apps installed and used and user's time-varying future apps usage behavior.

Subsequently, using Lyapunov optimisation, we develop an optimal control algorithm for identifying updates in user profiles and the user's usage behavior of actual apps in order to capture the temporal changes during profiling process. User profiles consist of various interests, gathered in similar categories, that are derived based on the user's (private) behavioral activity from utiilising installed apps, activities over web, and their interactions with ads. Our purpose is to protect attacks on privacy, via app-based profiling and ad-based inferencing, of selected private (that may be considered private by the user) interest categories in a user profile. E.g. the user may not wish the categories of gambling or porn to be presented in their profiles or to be targeted with relevant ads, which would of particular relevance when business devices are used for private purposes. In addition to privacy protection, which impose cost overhead by running newly suggested apps, the proposed framework minimises the cost introduced, termed as resource overhead, by bounding the use of new apps among various boundaries of weightages assigned to interests categories in user profiles.

Furthermore, we investigate the profiling process used by Google AdMob, a major analytics network, during establishing user profile and during when profile evolves with time, by investigating relation between mobile apps characteristics and the resulting profiles. We carry out extensive profiling experimentations by examining contribution of individual apps in a user profile, experiments with newly suggested apps for apps usage behavior protection along with evaluation of resource overheads, and evaluate effect of these experiments over user profiles; overall these experiments were run for over 5 months. Our experiments show that the mapping of interests interests categories can be predicted from installed and used apps with an accuracy of $81.4 \%$ along with the private and dominating interests categories based on user's activity over mobile device. In addition, we found that the profiling interests are selected from pre-defined set of interests categories and that the apps to interests mapping is deterministic, which requires specific amount of time (up to 72 hours) and certain level of activity to establish a user profile.

We propose various changes in 'User Environment' in a mobile advertising ecosystem i.e. we suggest 'System App' with following functionalities: It implements local user profiling based on apps usage and user's behavior, brows- ing history/searches and user's interactions with ads; 'System Engine' that keeps local repository and determines newly suggested apps; protects user's privacy for their private sensitive attributes; Implements proposed online control algorithm for jointly optimising user privacy and associated cost. Furthermore, we implement a POC (Proof Of Concept) 'System App' of our proposed framework to demonstrate its applicability in an actual environment. As opposed to legacy advertising operations where users are tracked for their activities, the 'System App' passes anonymous apps usage info/statistics and generated profile to the analytics servers within advertising system. In addition, the analytics server in current advertising system only evaluates stats for apps usage, which are recoded for the purpose of billing both for ad system and apps developers.

Finally, we discuss the use of other privacy protection mechanisms e.g., differential privacy, cryptographic approaches etc., in the mobile advertising environment and compare the proposed framework with these privacy protection mechanisms for various performance parameters.

The paper is organised as follows: Section 2 presents background on user profiling and the ads ecosystem, proposed addressed problem, and threat model. In Section 3, we present system model and investigate the profile creation and user profiling process. Section 4 presents optimal privacy preserving system and further discusses the proposed framework. The optimal control algorithm is discussed in Section 5. Various evaluation metrics are discussed in Section 6. Section 7 discusses system evaluation, our experimental setup and results. We further discuss the applicability of proposed framework and its comparison with other privacy protection mechanisms in Section 8. Related work is presented in Section 9 and conclusion in Section 10.

\section{Problem Formulation}

The Advertising and Analytic (A\&A) companies rely on users' tracking data to profile and to target them with relevant advertisements, both 'Web \& App'-based to cover vast majority of audience of diverse interests, to increase their advertising market revenue. This exposes sensitive information about users, in particular, their online behavior e.g., web brwosing/serching, or when profiling is based on apps representing sensitive and damaging information, e.g., gambling problems indicated by a game app, or the mobile apps usage behaviors e.g. playing games or use of gambling apps early morning in bed or during lunch break in office hours. Following, we present the privacy issues related to profiling process for both 'Web \& App' activity and app usage behavior, subsequently, we present the problem and threat model discussed in this paper.

\subsection{User profiling}

The advertising companies, e.g., Google, profile users based on information a user adds to the Google account, its estimation of user's interests based on the mobile apps usage and web histories, and data collected from the partnering analytics companies with Google, which effectively carry out targeted adverting based on the personal information extracted through various tracking technologies. 
Figure 1 shows an example user profile ${ }^{4}$, estimated by Google ${ }^{5}$, which consists of demographics (e.g. gender, ageranks) and profiling interests e.g. Action \& Adventure Films. We call this an Interests profile with all those interests defined by A\&A companies (e.g. Google) and is used by them to individually characterise user's interests across the advertising ecosystem. Similarly, we introduce Context profile that is the combination of apps installed from various categories, e.g. Games, Entertainment, Sports etc., on a user mobile device; detailed discussion over Context profile is given in Section 3. We note, using our extensive experimentations [4], that Context profile profile is also (partly) used by the analytics companies to individually characterise user's interests across the advertising ecosystem.

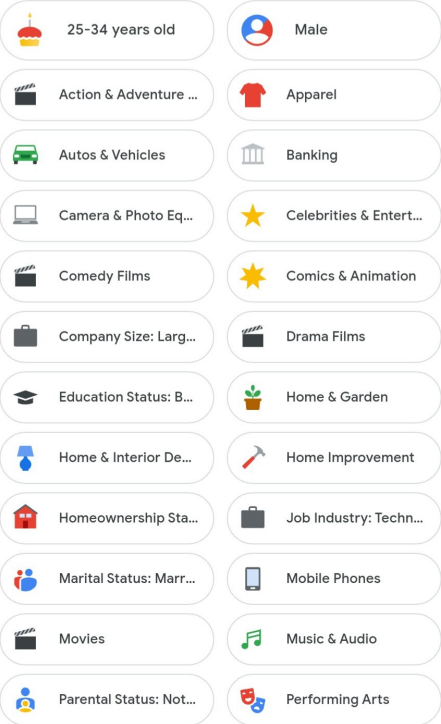

Fig. 1: An (anonymous) example user profile estimated by Google as a results of 'Web \& App' activity.

Furthermore, the ads targeting is based on demographics so as to reach a specific set of potential customers that are likely to be within a specific age range, gender etc. Google ${ }^{6}$ presents a detailed set of various demographic targeting options. The demographics are usually grouped into different categories, with specific options such as age-ranges, e.g. '1824', '25-34' etc., and gender e.g., 'Male', 'Female', and other demographic targeting options e.g. parental status, location etc. We note that this profiling is the result of interactions of user devices with AdMob SDK [4] that communicates with Google Analytics for deriving user profiles. A complete set of 'Web \& App' activities of individual user can be found under 'My Google Activity' ${ }^{7}$, which helps Google make services more useful.

Figure 2 shows various sources/platforms that Google uses to collect data and target users with personalised ads.

4. Google AdMob profile can be accessed through the Google Settings system app on Android-based devices; accessible through Google Settings $\rightarrow$ Ads $\rightarrow$ Ads by Google $\rightarrow$ Ads Settings.

5. Profiling interests can be found here: https://adssettings.google.c om/authenticated.

6. Demographic Targeting: https://support.google.com/google-ad s/answer $/ 2580383$ ?hl=en

7. https://myactivity.google.com/myactivity?otzr=1
These include a wide range of different sources enabled with tracking tools and technologies, e.g., the 'Web \& App' activities are extracted with the help of Andoird/iOS SDKs, their interactions with Analytics servers within the Google network, cookies, conversation tracking ${ }^{8}$, web searches, user's interactions with presented ads etc. Similarly, Google's connected home devices and services ${ }^{9}$ rely on data collected using cameras, microphones and other sensors to provide helpful features and services ${ }^{10}$. The tracking data (up to several GBs of data), personalised for individual users, can be exported using Google Takeout ${ }^{11}$ for backup or use it with a service outside of Google. This includes the data from a range Google products, such as, email conversations (including Spam and Trash emails), contacts, calendar, browsing and location history, and photos.

\subsection{Entities within advertising system}

Figure 3 shows a representative in-app mobile advertising ecosystem showing the information flow among its different parties for profiling users for enabling targeted ads based on 'Web \& App' activities. User usually install a number of apps on their mobile devices, that are utilised with specific frequency. The mobile apps include analytics SDK, which directly reports user's activity (as mentioned in above section) and sends ad requests to the analytics and ad network. Various advertising entities play important role in enabling tracking and ads dissimilation in an ad system, comprises the Aggregation, Analytics, Billing, and the Ads $\mathrm{Pl}$ lacement servers. Collected tracking data is used by the Analytics server that constructs Interests profiles (associated with specific mobile devices and corresponding users) with specific profiling interests related to user's (private) behavior. The targeted ads are served to mobile users according to their (individual) profiles. We note that other i.e., generic ads are also served [14]. The Billing server includes the functionality related to monetising Ad impressions (i.e. ads displayed to the users in specific apps) and Ad clicks (user action on presented ads).

\subsection{Problem Statement}

The problem addressed in this paper is where the A\&A companies track mobile users for their activities, profile them (inferred via relationships among individuals, their monitored responses to previous advertising activity and temporal behavior over the Internet) and target them with ads specific to individual's interests. The user profiling and ads targeting expose sensitive information about users [1], [4], e.g. the target could browse through medical related websites or apps, revealing (including a third-party, such as the website owner) to the advertising systems that the user has medical issues.

Furthermore, we address the privacy issues where an adversary (either the analytics companies examining user

8. https://support.google.com/google-ads/answer/6308

9. Google's Connected Home Devices and Services: https://support. google.com/googlenest/answer/9327662? p=connected-devices\&visit_ id $=637357664880642401-2675773861 \& \mathrm{rd}=1$

10. Sensors in Google Nest devices: https://support.google.com/g ooglenest/answer/9330256?hl=en

11. Your account, your data: https://takeout.google.com/ 


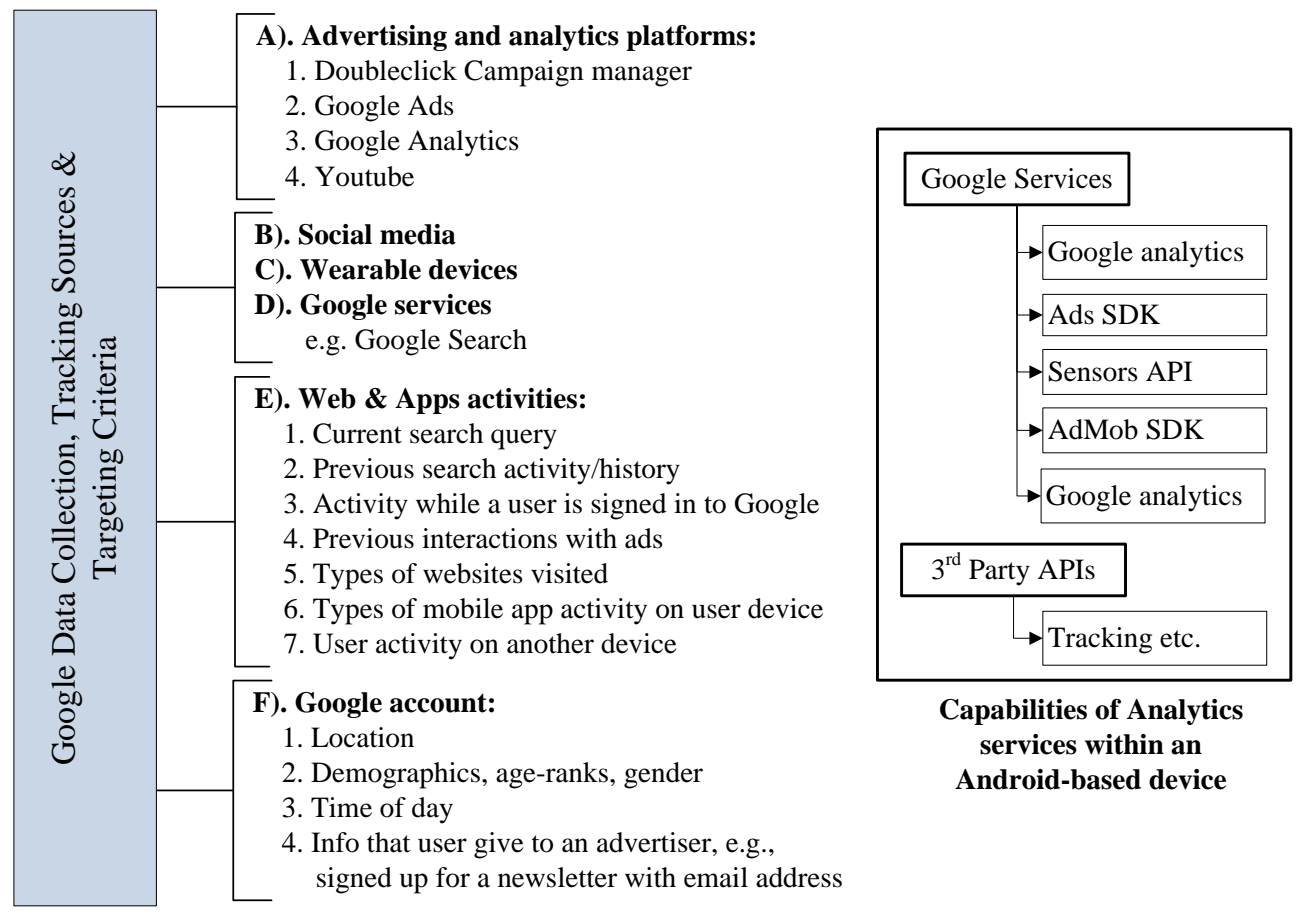

Fig. 2: Google's data collection and tracking sources for targeting users with personalised ads (left) and tracking capabilities of analytics libraries enabled within mobile devices (right).

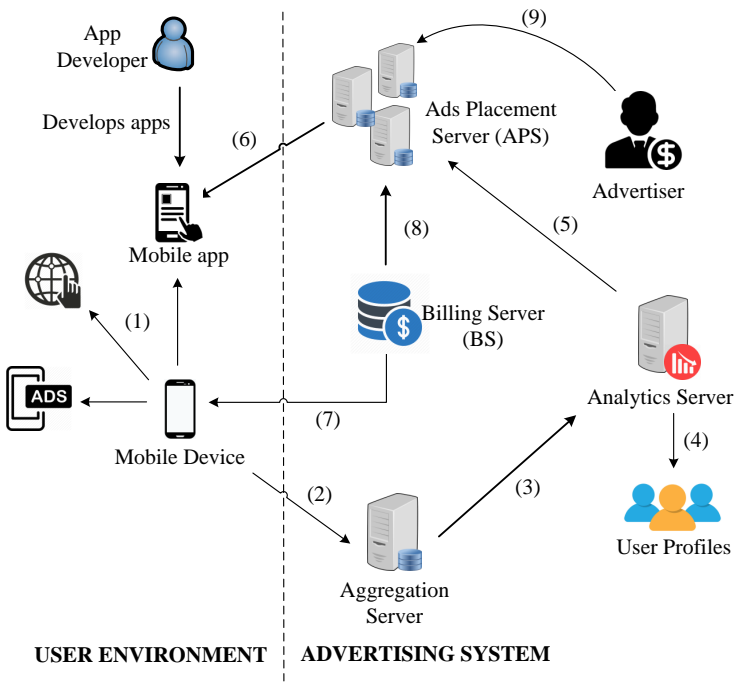

Fig. 3: The advertising system consists of user and advertising environments. Following functionalities: (1) Data collection and tracking, (2) Send tracking data to Aggregation server, (3) Forward usage info to Analytics server, (4) User profiling, (5) Send profiling info to APS, (6) Deliver targeted/generic ads, (7) Billing for Apps Developer, (8) Billing for Ad System, (9) Advertiser uploads ads, who wishes to advertise with Ad system.

activity or an intruder listening to the ad or control traffic) can determine the app's usage activity e.g., someone plays games during late night or early morning and their activity is intercepted by their neighbors. The user's apps usage activities can be exposed by intercepting the apps communi- cation using the connected network [12], in addition, users' activities are also exposed to the advertising systems during their interactions using ad/analytics SDKs. Figure 4 shows an example apps usage profile for a typical day, showing a direct threat to user's privacy for apps usage.

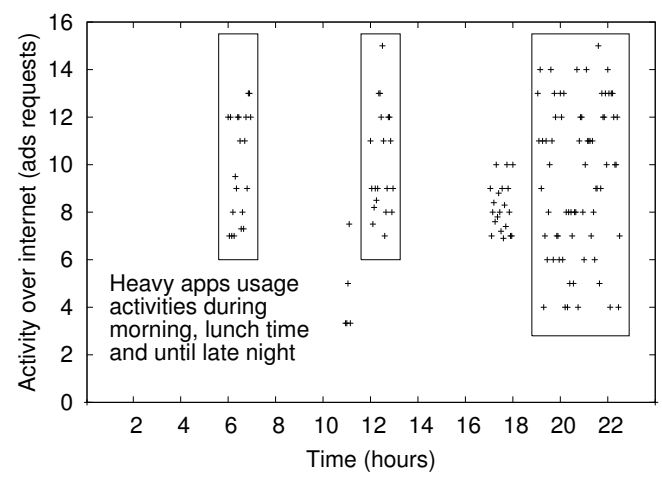

Fig. 4: Apps usage profile for typical day showing apps usage activity during 24 hours.

In particular, we address three privacy attacks: 1. Legitimate user profiling by $\mathrm{A} \& \mathrm{~A}$, the user profiling is implemented via an analytics SDK for reporting user's activities to A\&A companies, hence, intercepts various requests to/from mobile users. 2. Indirect privacy attack, involves third parties that could intercept and infer user profiles based on targeted ads. 3. Apps usage behavioral attack to know user's apps usage activities. In the first two privacy attacks, we presume that the users do not want to expose their private interests to adversaries (including advertising agencies) and are willing to receive relevant ads based on their interests. 


\subsection{Threat Model}

Our primary goal in this work is to achieve an optimal privacy preserving profile management that preserves user's privacy due to profiling interests derived via apps usage, privacy in terms of apps usage behavior, users' web history/searches, and their interactions with ads. We start by analysing and developing the profiling process, identify the dominating interests that expose user privacy and could affect ads targeting, the proportion of interests in user profile, and the apps usage activity. We further compare proposed solution and its applicability with other privacy preserving mechanisms such as differential privacy, anonymisation, randomisation, Blockchainbased solutions and crypto-based mechanisms e.g., private information retrieval) in an advertising scenario for the problems addressed in this work. Finally, we evaluate the trade-off between the achieved user privacy, cost of achieving privacy and targeted ads. Hence, in this paper, we jointly optimise the user's privacy due to profiling interests, the cost of achieving privacy, and the apps usage behavior.

\section{System Components}

We formalise the system model that consists of the apps' profiles, interests' profiles and the conversion of resulting profiles by use of applications in an app profile. In particular, we provide the insights of establishment of Interests profiles by individual apps in the Context profiles and then show how the profiles evolve when some apps other than the initial set of apps are utilised.

\subsection{System Model}

We denote an app by $a_{i, j}, i=1, \ldots, A_{j}$, where $A_{j}$ is the number of apps that belong to an app category $\Phi_{j}$, $j=1, \ldots, \phi$ and $\phi$ is the number of different categories in a marketplace (e.g., in Google Play or in the Apple App store). An individual app $a_{i, j}$ is characterised by a set of keywords $\kappa_{i, j}$ that includes app's category (e.g. Business, Entertainment etc.) and dictionary (specific to this particular app) keywords from its description. We represent with $\mathcal{A}$ the entire set of mobile apps in any marketplace, organised in various categories.

A user may be characterised by a combination of apps installed on their mobile device(s), comprising a subset $S_{a} \in$ $\mathcal{A}$. Following, the Context profile $K_{a}$ can be defined as:

$$
K_{a}=\left\{\left\{a_{i, j}, \Phi_{j}\right\}: a_{i, j} \in S_{a}\right\}
$$

The A\&A companies, such as, Google or Flurry, partly profile and target users based on the combination of mobile apps installed on their devices i.e. Context profile. We have the following constraints on the user's Context profile:

$$
\begin{array}{r}
0<n\left(K_{a}\right) \leq \mathcal{A} \\
n\left(K_{a}\right) \geq 1 \\
\Phi_{j}\left(a_{i, j}\right) \neq 0 \\
\phi \subseteq \Phi_{j} ; \forall j
\end{array}
$$

The $n\left(K_{a}\right)$ is the total number of apps installed on a device, hence, using Eq. (3), it is important to make sure the $n\left(K_{a}\right)$ should not exceed the total number of available apps; that a mobile device must have at least one app installed for contextual targeting; that an app $a_{i, j}$ must belong to any of the specified categories; and that the app's category is not undefined within an app market. Various important notations and their descriptions are presented in Table 1.

\subsection{Representing apps in user profile}

We note that the A\&A companies classify users by defining a set of profiling interests $\mathcal{G}$, i.e., characteristics that may be assigned to users i.e. an Interest profile. E.g. Google profile interests ${ }^{12}$ are grouped, hierarchically, under various interests categories, with specific interests. We denote, using $g_{k, l}, k=1, \ldots, G_{l}$, where $G_{l}$ is total number of interests that belong to an interest category $\Psi_{l}, l=1, \ldots, \psi \cdot \psi$ is total number of interest categories defined by analytics companies. An individual interest $g_{k, l}$ consists of a set of keywords $\kappa_{k, l}$, which characterises specific interests. Following, to enable interest targeting, represents an Interests profile $I_{g}$, consists of a subset $S_{g}$ of specific interests:

$$
I_{g}=\left\{\left\{g_{k, l}, \Psi_{l}\right\}: g_{k, l} \in S_{g}\right\}
$$

Although various types of information may be used to generate user profiles, as shown in Figure 2, however, our focus is mainly on installed and used/unused apps ${ }^{13}$, the history resulted from web browsing or web searches, and the clicked ads i.e. collectively described as the 'Web \& Apps' activity. Similarly, other targeting criteria can also be represented in an Interest profile as a specific interest e.g. demographics, contributing analytic platforms, social media, and other Google services. An example demographics interests is also shown in Figure 1.

The targeting components are eventually grouped in Interest profile that are used for ads targeting. The Interest profiles undergo different processes e.g. the profile establishment process (i.e. the derivation of Interests profile by apps; $K_{a} \rightarrow I_{g}$ ) (generating a specific set of interests $S_{K_{a}}\left\{\Psi_{l}\right\}_{l=1}^{\varepsilon}$ using a mapping function $f$ i.e. $\left.f: \Phi_{j} \rightarrow \Psi_{l}=S_{K_{a}}\left\{\Psi_{l}\right\}_{l=1}^{\psi}\right)$, the profile evolution $I_{g}^{\prime}$ process (i.e. each time variations in the users' behaviour are observed), and profile development process (i.e. the minimum level of activity required to develop a stable user profile $I_{g}^{f}$ ) i.e. $I_{g}^{f}=I_{g} \cup I_{g}^{\prime}$. Figure 5 shows user profiling process, detailed discussion over these various processes of user profiling can be found in [15]. Furthermore, detailed experimental evaluations over insights on profiling rules, apps usage threshold during profiling establishment, and mapping rules for Context profile to Interest profiles can be found in [4], [16].

In order to represent part (along with the dominating interest categories) of each interest category, we assign weightage to each category $\Psi_{l}$ present in the final user profile $I_{g}^{f}$; represented as $\eta_{l}\left(\Psi_{l}\right)$, under the following two constraints:

12. Google profile interests are listed in https://adssettings.goog le.com/authenticated?hl=en, managed under the 'How your ads are personalized'; other options and Google services can also be verified on Google Dashboard https://myaccount.google.com/dashboard?hl=en.

13. The list of installed apps on an Android based phone can be withdrawn using following shell command: adb shell pm list packages. The full list of installed apps is included in the library. do file /data/data/com.android.vending/databases/library.db. 


\begin{tabular}{|c|c|}
\hline Symbols & Description \\
\hline $\mathcal{A}$ & Available apps in a marketplace \\
\hline$\phi$ & Total number of apps categories, $\Phi_{j}$ is a selected category, $j=1, \ldots, \phi$ \\
\hline$S_{a}$ & Subset of apps installed on a user's mobile device \\
\hline$a_{i, j}$ & An $a p p a_{i, j} \in A, i=1, \ldots, A_{j}, j=1, \ldots, \phi, A_{j}$ is the number of apps in $\Phi_{j}$ \\
\hline$\kappa_{i, j}$ & Set of keywords associated with individual $a p p a_{i, j}$ including its category \\
\hline$K_{a}$ & App profile consisting of apps $a_{i, j}$ and their categories $\Phi_{j}$ \\
\hline $\mathcal{G}$ & Set of interests in Google interests list \\
\hline$\Psi_{l}$ & Interest category in $\mathcal{G}, l=1, \ldots, \psi, \psi$ is the number of interest categories defined by Google \\
\hline$S_{g}$ & Subset of Google interests in $\mathcal{G}$ derived by $S_{a}$ \\
\hline$I_{g}$ & Interest profile consisting of $g_{k, l}, g_{k, l} \in S_{g}$ \\
\hline$g_{k, l}$ & An interest in $I_{g}, k \in G_{l}, l \in \psi$ \\
\hline$\kappa_{k, l}$ & Set of keywords associated with individual interest $g_{k, l}$ including interest category \\
\hline$S_{g}^{i, j}$ & Set of interests derived by an $a p p a_{i, j}$ \\
\hline$f$ & Mapping that returns the derivation of apps (or history, ad's category) category to interest category \\
\hline$\eta_{l}\left(\Psi_{l}\right)$ & The weightage assigned to interest category in $\Psi_{l}$ in a profile with, respectively with $\eta_{l}^{\min }$ and $\eta_{l}^{\min }$ thresholds \\
\hline$\Pi_{m}$ & Profiling components for representing browsing history/searches \\
\hline$\Gamma_{n}$ & Profiling components for representing interactions with ads \\
\hline$t$ & Time slot \\
\hline$C_{t}\left(\eta_{l}\left(\Psi_{l}\right)\right)$ & Tracking change in interest category due to changes in apps usage \\
\hline$C_{t}\left(\eta_{m}\left(\Pi_{m}\right)\right)$ & Tracking change in web browsing history/searches \\
\hline$C_{t}\left(\eta_{n}\left(\Gamma_{n}\right)\right)$ & Tracking interactions with ads \\
\hline$U^{\prime \tau}\left(K_{a}\right)$ & Usage of apps in $K_{a}$ at time slot $t$; average usage of each app is given by $\overline{U^{\prime \tau}\left(K_{a}\right)}$ \\
\hline$\eta_{l^{\prime}}\left(\Psi_{l^{\prime}}\right)$ & The weightage assigned to interest category generated by newly suggested app(s), where $l^{\prime} \neq l$ \\
\hline$S_{o}$ & Set of newly suggested apps \\
\hline$C^{t}$ & Reduction of overall user's time available by use of newly suggested apps to the original apps \\
\hline$R^{t}$ & $\begin{array}{l}\text { Resource usage of newly suggested apps, in particular, } R_{b}^{t}, R_{c}^{t}, R_{p}^{t} \text { respectively represent the battery consumption, } \\
\text { communication, and processing resource usage }\end{array}$ \\
\hline$\beta$ & Adjustable parameter to achieve trade-off between user privacy and targeted ads \\
\hline$R_{l}^{t}$ & An advertising request reported at $t$ via ad/analytics SDK e.g. an ad request for display/ad click \\
\hline$\varepsilon$ & Adjustable parameter to achieve apps usage to preserve privacy of user's app usage behavior \\
\hline$p(t)$ & The penalty for minimising the upper bound on $C^{t}$, profiling privacy, and apps usage behavior \\
\hline
\end{tabular}

TABLE 1: List of Notations

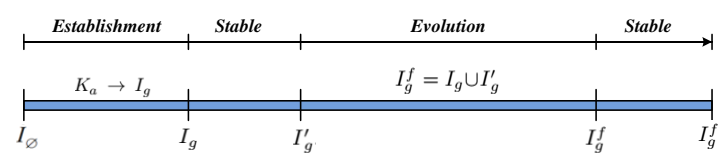

Fig. 5: Profile establishment \& evolution processes. $I_{\emptyset}$ is the empty profile before any activity over user device. During the stable states, the Interest profiles $I_{g}$ or $I_{g}^{f}$ remains the same and further activities of the same apps have no effect over the profiles [15].

$$
\begin{array}{r}
\eta_{l}^{\min } \leq \eta_{l}\left(\Psi_{l}\right) \leq \eta_{l}^{\max } \quad \forall l \in \Psi_{l} \mid l \in \psi \\
0<\eta_{l}\left(\Psi_{l}\right) \leq \eta_{l}^{\max }
\end{array}
$$

Hence, weightage given to an interest category $\eta_{l}\left(\Psi_{l}\right)$ is within the $\eta_{l}^{\min }$ and $\eta_{l}^{\max }$ threshold weightages. Subsequently, the user profile is represented as, $I_{g}=\eta_{l}\left(\Psi_{l}\right), \forall l \in$ $\psi$.

We note, using our extensive profiling experimentations [4], [16], that the set of installed apps, on a mobile device, do not necessarily contribute during profiling, in which case specific apps do not draw any interests i.e. an empty set. In addition, Eq. (5) ensures that the assigned threshold should be non-negative and cannot exceed the maximum threshold within a user profile.

Example - Assigning weightages: Let a user has five mobile apps installed on her device i.e. three from category $a$ and one from category $b$ and $c$ each, respectively with the following percentage of usage time ${ }^{14}: a=20 \%, b=70 \%, c=$ $10 \%$; for simplicity we do not consider apps that are installed but not used, which can be assigned the lowest weightages e.g. $1 / n\left(K_{a}\right)$ including system installed apps. The weightage for each category $\eta_{l}\left(\Psi_{l}\right)$ can be evaluated as follows: $\eta_{l}(a)=3 / 5+20 / 100=0.8 ; \quad \eta_{l}(b)=1 / 5+70 / 100=0.9 ;$ $\eta_{l}(c)=1 / 5+10 / 100=0.3$. Hence, it requires that the user is supposed to be targeted with the ads related to category $b$ with highest proportion, followed by $a$ and $c$. Note that for simplicity, these weightages can be normalised within the range $0-1$; subsequently, the proportion for delivery of targeted ads would be: $a=0.40, b=0.45$, and $c=0.15$.

\subsection{Representing browsing history/searches and ad- interactions in a user profile}

We respectively represent $\Pi_{m}$ and $\Gamma_{n}$ profiling components as user history/search and ad-interactions (e.g. ad click). Subsequently, we assign weightages to both these components:

$$
\begin{gathered}
\left(\eta_{l}^{\min }, 0\right)<\eta_{m}\left(\Pi_{m}\right) \leq \eta_{l}^{\max } \quad \forall m, \forall l \in[1, \psi] \\
\left(\eta_{l}^{\min }, 0\right)<\eta_{n}\left(\Gamma_{n}\right) \leq \eta_{l}^{\max } \quad \forall n, \forall l \in[1, \psi]
\end{gathered}
$$

Note that the minimum threshold, on the left sides of Eq. (6) \& (7) i.e. taken as a minimum of ' $\eta_{l}^{\min }\left(\Psi_{l}\right)$ ' and $' 0$ ', to respectively represent its weightage as higher than the

14. Use adb logcat $-v$ threadtime on an Android-based device to find the running time of an app; threadtime (default) is used to display the date, invocation time, tag, priority, TID, and PID of a thread issuing the message. 
minimum weightage of present interests (and hence to show its importance in current targeting components) or lower its dominating factor to slightly higher than ' 0 '.

Subsequently, we have the following equivalent user profile, as a representation of weightage of all the targeting criteria,

$$
I_{g}=\eta_{l}\left(\Psi_{l}\right)+\eta_{m}\left(\Pi_{m}\right)+\eta_{n}\left(\Gamma_{n}\right)
$$

\subsection{Profile updating}

An important factor in ads targeting is to track user's activity to find temporal changes in a user profile; hence, the profile and ads targeting is updated each time variations in user behavior is observed i.e. the targeting criteria result in interests other than the existing set of interests. We note that following criteria (but not limited to) is used to track changes in a use profile: installation/un-installation of an app i.e. a user uses new set of apps $S_{a}^{\prime}$, which has no overlap with the existing set of apps $S_{a}$, increase/decreases of use of an existing app, start use of an 'un-used' app, interactions with ads, and changes in web browsing history/searches. Let $\eta_{l}^{\prime}$ min and $\eta_{l}^{\prime} \max$ are new minimum and maximum threshold due to changes in apps usage, then following conditions may hold (provided that the usage time is i.i.d i.e. independent and identical distributed, with some unknown probability distribution):

$$
\begin{aligned}
& \eta_{l}^{\prime \max }>\eta_{l}^{\max } ; \eta_{l}^{\prime}{ }^{\min }>\eta_{l}^{\min } \rightarrow \text { apps uninstalled } \\
& \eta_{l}^{\prime}{ }^{\max }<\eta_{l}^{\max } ; \eta_{l}^{\prime}{ }^{\min }<\eta_{l}^{\min } \rightarrow \text { new apps installed } \\
& \eta_{l}^{\prime}{ }^{\max }<\eta_{l}^{\max } ; \eta_{l}^{\prime \min }<\eta_{l}^{\min } \rightarrow \text { unused apps become used } \\
& \eta_{l}^{\prime}{ }^{\max }>\eta_{l}^{\max } ; \eta_{l}^{\prime}{ }^{\min }>\eta_{l}^{\min } \rightarrow \text { used app become unused }
\end{aligned}
$$

Based on above constraints, we have the following equivalent constraint due to changes in app activity:

$$
\begin{gathered}
\eta_{l}^{\prime} \min \left(\Psi_{l}\right) \geq \min \left\{\eta_{l}^{\min }, \eta_{l}^{\prime}{ }^{\min }\right\} \\
\eta_{l}^{\prime} \max \left(\Psi_{l}\right) \geq \max \left\{\eta_{l}^{\max }, \eta_{l}^{\prime} \max \right\}
\end{gathered}
$$

In addition, such changes in browsing history/searches and interactions with ads can also be expressed with threshold limits, as explained in next section.

\subsection{Profile evolution}

Furthermore, subsequent changes in a user profile (w.r.t. time $t)$ are represented as $C_{t}\left(\eta_{l}\left(\Psi_{l}\right)\right) ; C_{t}\left(\eta_{m}\left(\Pi_{m}\right)\right)$ and $C_{t}\left(\eta_{n}\left(\Gamma_{n}\right)\right)$ respectively for changes in apps usage, web browsing history/searches and interactions with ads. Hence, Eq. (8) can be re-written as:

$$
I_{g}^{t}=C_{t}\left(\eta_{l}\left(\Psi_{l}\right)\right)+C_{t}\left(\eta_{m}\left(\Pi_{m}\right)\right)+C_{t}\left(\eta_{n}\left(\Gamma_{n}\right)\right)
$$

Since the assigned weightages should always be nonnegative and bounded by maximum thresholds (i.e. maximum convergence point in specific period e.g. of every 24 hours), hence we need to make sure the following:

$$
\begin{array}{r}
0<C_{t}\left(\eta_{l}\left(\Psi_{l}\right)\right) \leq C_{t}^{\max }\left(\eta_{l}\left(\Psi_{l}\right)\right) ; \forall t, \forall l \in[1, \psi] \\
0<C_{t}\left(\eta_{m}\left(\Pi_{m}\right)\right) \leq C_{t}^{\max }\left(\eta_{m}\left(\Pi_{m}\right)\right) ; \forall t \\
0<C_{t}\left(\eta_{n}\left(\Gamma_{n}\right)\right) \leq C_{t}^{\max }\left(\eta_{n}\left(\Gamma_{n}\right)\right) ; \forall t
\end{array}
$$

As mentioned earlier, the other profiling components, such as history/searches and interactions with ads, are also mapped to profiling interests $\Psi_{l}$, to produce a unified profile with different dominating interest categories, e.g. for adinteractions: $f: \Gamma_{n} \rightarrow \Psi_{l}=S_{K_{a}}\left\{\Psi_{l}\right\}_{l=1}^{\psi}$. We presume that all changes in user profiles are distributed with an unknown probability distribution and that these profile weightages are deterministically bounded by a finite constant e.g. $C_{t}^{\max }\left(\eta_{m}\left(\Pi_{m}\right)\right)$ for web history, so that (incorporated in user profile) at time slot ' $t$ ', Eq. (12) can be re-written, as a unified profiling interests, as:

$$
I_{g}^{t}=\sum_{\forall t} C_{t}\left(\eta_{l}\left(\Psi_{l}\right)\right) ; \quad \forall l \in\{[1, \psi], m, n\}
$$

Similarly, the newly updated (or change in existing) interest categories are reflected in profile at ' $t+1$ ' as: $I_{g}^{t+1}=I_{g}^{t}+C_{t+1}\left(\eta_{l}\left(\Psi_{l}\right)\right)$. For $n$ amount of time:

$$
0<I_{g}^{t+1} \leq I_{g}^{t+n} \quad \forall t
$$

$I_{g}^{t+n}$ is the maximum convergence point of a user profile i.e. the point where users are targeted with the most relevant ads. We envisage that this information is regularly updated, e.g. once per 24 hours, which we call evolution threshold i.e. the time required to evolve profile's interests, and is used to reflect the updated profile and fetch associated targeted ads:

$$
\lim _{t \rightarrow \infty} \sum_{\tau=1}^{t} I_{g}^{\tau}
$$

Recall that profile at this stage, as $t \rightarrow \infty$, due to maximum profile convergence, it contains the dominating interests $g_{k, l}$ that are considered private by user, and hence exposes user's privacy. Our goal is to design a control algorithm that jointly optimises user's privacy (both profiling and app usage activity at different time of the day/night, as detailed in next section) and cost of newly (suggested) apps (discussed in Section 4).

\subsection{Apps usage profile}

Let $U^{t}\left(K_{a}\right)$ represents the apps usage at time slot $t$ (during day/night, note, for simplicity we do not consider the app usage time for individual apps). The apps usage behavior for every user is time-varying, e.g. some users play game/gambling apps during the lunch time or early in bed when she gets up, or an employee might scroll through the stocks in a broker's application during lunch break, to be exposed to her employer. We note that the user's apps usage activity can be intercepted from the connected network [12] or through $a p p$ 's interactions with the advertising systems [4], [14], [15]. Let $\lim _{t \rightarrow \infty} \sum_{\tau=1}^{t} U^{\tau}\left(K_{a}\right)$ denotes app's usage at different time slots. The app's usage time varies during the 24 hours for each user, which also exposes user's privacy (irrespective of the category of $a p p^{\prime}$ s usage) in terms of use of apps during various times of the day/night. 


\section{Optimal Privacy Preserving Profiling}

Based on the above requirements for user profiling, we now study optimal privacy preserving profile management that is cost effective, preserve user's privacy due to profiling interests and the apps usage behavior, in addition to, user's web history/searches and interactions with ads.

\subsection{Proposed framework}

Figure 6 presents detailed overview of the proposed framework, introducing changes to 'User Environment' that implements local user profiling both for Context profile and Interests profiles and further protects their privacy, presented in Section 4.2, similarly, preserves user's privacy for user's apps usage behavior during day/night time, detailed in Section 4.3, implemented via 'System App'. We implement these various functionalities via a Proof of Concept (PoC) mobile app; detailed discussion is given in Section 7.1. The 'System App' also implements this framework that jointly optimises the profiling process and preserves user privacy in a cost-effective way, as detailed in Sections 4.4, 4.5, and 4.6. We suggest that this framework can be integrated into AdMob SDK since the current ad ecosystem carries out user profiling and targeted ads via SDKs, which will require SDK modifications.

\subsection{Protecting sensitive profiling interests}

To protect the private interest categories i.e. sensitive to users, we select various other apps based on similarity metric $[4],[16]^{15}$ to reduce the dominating private interest categories present in a user profile. This metric is calculated based on app keywords $\kappa_{i, j}$ using the $t f-i d f$ (cosine similarity) metric [14]. This strategy is not metric-specific hence other similarity metrics e.g., jaccard index, can also be used.

The newly selected candidate apps, which we call the $o b$ fuscation apps (note that in this work, we interchangeably use both 'newly suggested apps' and 'obfuscation apps'), are selected (and run for specific amount of time, as described in Eq. (23), (24), and (25)) from apps categories $\Phi_{j}, j=1, \ldots, \phi$ other than the private category $\Phi_{p} ; \Phi_{p}$ is considered private by users that they want to protect. The newly suggested set $S_{o}$ comprises those apps with highest similarity to the existing set of apps i.e. $S_{a}$. In addition, the user may protect any number of private interests categories $\Psi_{p}, p=1, \cdots, \Omega$ and $\Omega$ is the set of interests categories that are private to the user. Furthermore, we presume that the selected app(s) will always generate the profiling interests other than the private profiling interest(s) i.e. $l^{\prime} \neq l$.

We assign weightage $\eta_{l^{\prime}}\left(\Psi_{l^{\prime}}\right)$ to the newly generated profiling interests, $I_{g}^{\prime} t$, in order to reflect its effect over 1. privacy (i.e. disruption in user profile) and 2. targeted ads (disruption in receiving targeted ads based on private profiling interests, exposed to ad/analytics networks):

$$
\eta_{l}^{\min }\left(\Psi_{l}\right)>\eta_{l^{\prime}}\left(\Psi_{l^{\prime}}\right)>0
$$

15. Detailed discussion over profile obfuscation to achieve user privacy in an app-based user profiling, using various obfuscation strategies, can be found in [4], [16].
Cost is usually defined as the ratio of obfuscating to original data [17]. We further elaborate on Cost in Section 6.1.2. Recall that, beyond privacy protection, the use of these newly suggested apps is used to protect user privacy of apps usage behavior e.g., during the 24 hours period.

\subsection{Control objective for apps usage behavior}

As mentioned in Section 3.6, intuitively speaking, in order to achieve user privacy for apps usage at different time slots $t$ of the day, the apps usage profile $U^{t}\left(K_{a}\right)$ needs to be 'flatten' as $t \rightarrow \infty$, as much as possible, by running additional (newly suggested apps, as described in above section) apps i.e. $U^{\prime} t\left(K_{a}\right)$. Subsequently, the profile becomes:

$$
I_{g}^{\prime t}=I_{g}^{t}+U^{\prime t}\left(K_{a}\right)
$$

Let $\overline{U^{t}\left(K_{a}\right)}=\lim _{t \rightarrow \infty} \frac{1}{t} \sum_{\tau=1}^{t} U^{\tau}\left(K_{a}\right)$ represents the average apps usage for a user $K_{a}$, alternatively $\overline{U^{t}\left(K_{a}\right)}=$ $\overline{C_{t}\left(\eta_{l}\left(\Psi_{l}\right)\right)}, \forall l \in[1, \psi]$. In real time, at different time slots, the apps usage needs to be controlled with as little deviation from $\overline{C_{t}\left(\eta_{l}\left(\Psi_{l}\right)\right)}$ as possible. Note that this is also applicable to the interests in $I_{g}^{t}$ generated via browsing history/searches and interactions with ads, since, as mentioned earlier that $\Pi_{m} \rightarrow \Psi_{l}$ and $\Gamma_{n} \rightarrow \Psi_{l}$, in addition, using both these activities, the user devices interact with the ad/analytic networks for tracking user's activity along with their (device's) usage behavior.

Subsequently, the control objective is to minimise the variance of $\overline{U^{t}\left(K_{a}\right)}$, i.e.:

$$
\lim _{t \rightarrow \infty} \frac{1}{t} \sum_{\tau=1}^{t} \mathbb{E}\left\{\left(I_{g}^{\tau}-\overline{U^{\prime \tau}\left(K_{a}\right)}\right)^{2}\right\}
$$

The privacy protection in apps usage behavior, in addition to, preserve user's privacy due to profiling interests, can be achieved by using few other suggested apps, described in next section, for our proposed scenario.

\subsection{Objective function}

In this paper, we jointly optimise the user's privacy due to profiling interests, the cost of running obfuscation apps, and the apps usage behavior. The objective function can be expressed as:

$\lim _{t \rightarrow \infty} \sum_{\tau=1}^{t}(\begin{array}{l}\underbrace{C^{\tau} \eta_{l^{\prime}}\left(\Psi_{l^{\prime}}\right)}_{\text {Apps usage privacy }} \\ \begin{array}{l}\text { Cost of running obfs. apps } \\ \beta\left(I_{g}^{\tau}-\overline{U^{\prime \tau}\left(K_{a}\right)}\right)^{2}\end{array}\end{array}+\underbrace{\beta\left(I_{g}^{\tau}+\eta_{l^{\prime}}^{\tau}\left(\Psi_{l^{\prime}}\right)\right)}_{\text {Profiling privacy }}+$

Here, the $\beta$ parameter is selected by user in order to achieve a trade-off between user privacy and targeted ads. Note that the selection of this parameter affects the targeted ads as a result of disruption in a user profile. We describe the following various scenarios for introducing (along with the number of apps) newly selected apps: 


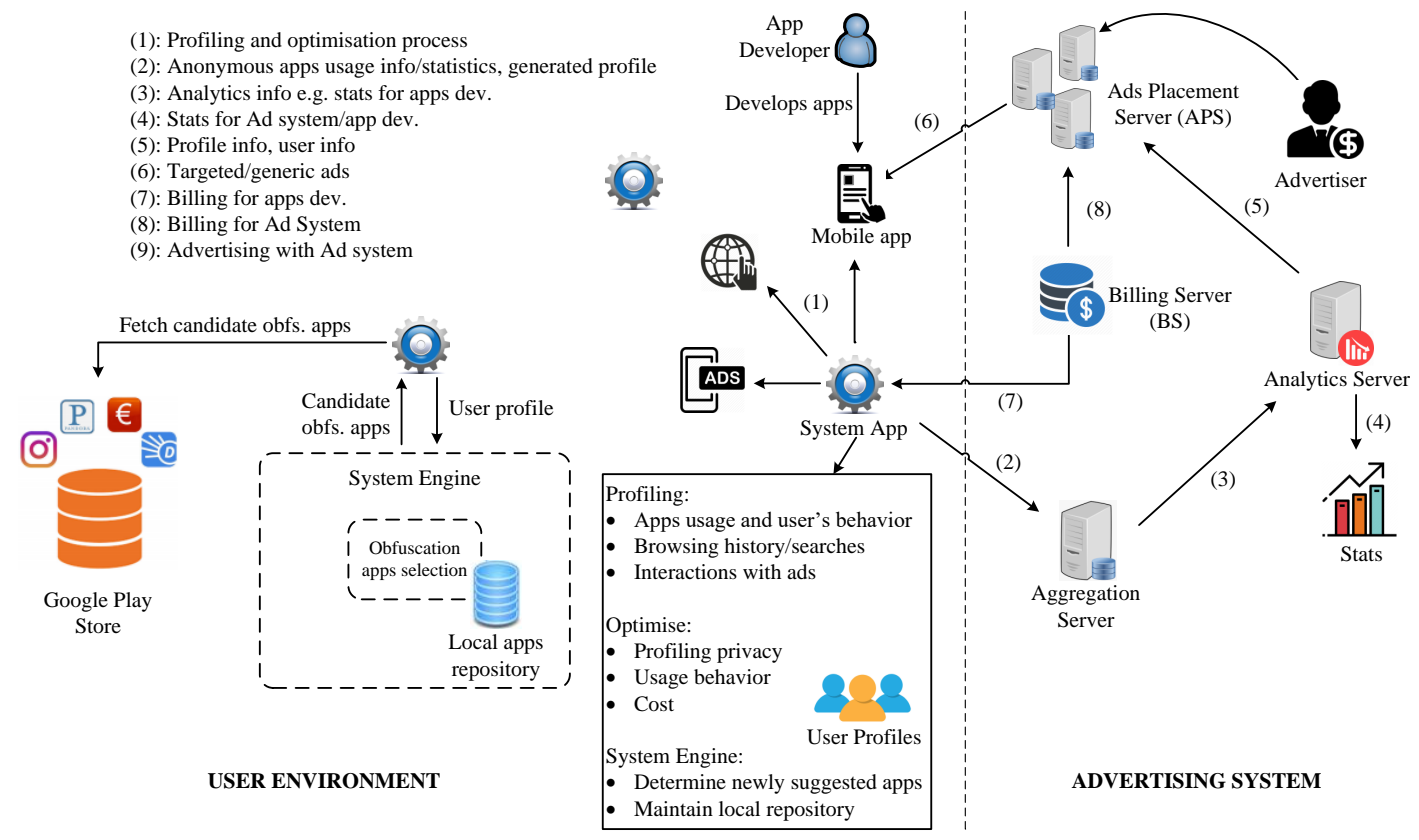

Fig. 6: The proposed advertising system with changes in user and advertising environments.

$$
\begin{array}{r}
0<\eta_{l^{\prime}}\left(\Psi_{l^{\prime}}\right) \leq \eta_{l}^{\min }\left(\Psi_{l}\right) \\
\eta_{l}^{\min }\left(\Psi_{l}\right) \leq \eta_{l^{\prime}}\left(\Psi_{l^{\prime}}\right) \leq \eta_{l}^{\max }\left(\Psi_{l}\right) \\
\eta_{l^{\prime}}\left(\Psi_{l^{\prime}}\right) \geq \eta_{l}^{\max }\left(\Psi_{l}\right)
\end{array}
$$

The first scenario (i.e. Eq. (23)) introduces newly obfuscation apps that would introduce minimum disruption in a user profile, hence, achieves lower privacy and attracts higher targeted ads, as opposed to the last scenario, which introduces highest disruption in a user profile i.e., achieves higher privacy and attracts less relevant targeted ads. On the other hand, the middle scenario introduces medium disruption in a user profile and achieves a balance between user privacy and targeted ads. An empirical example for various scenarios is given in Figure 7. We envisage that this scenario (24) will further introduce medium operating cost of the selected obfuscation apps i.e. $C^{\tau} \eta_{l^{\prime}}\left(\Psi_{l^{\prime}}\right)$.

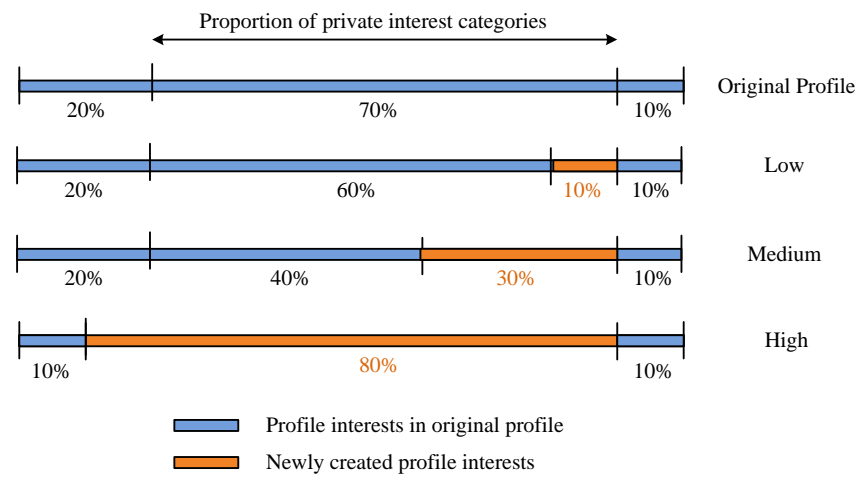

Fig. 7: Trade-off between privacy and targeted ads achieved via various obfuscation scenarios of Low, Medium, and High profile (interests), respectively introducing $10 \%, 30 \%$, and $80 \%$ disruption in a user profile.

\subsection{Problem formulation}

The optimal privacy preserving user profiling and targeted ads can be formulated as a dynamic optimisation problem:

$$
\min \lim _{t \rightarrow \infty} \sum_{\tau=1}^{t}\left(\begin{array}{l}
C^{\tau} \eta_{l^{\prime}}\left(\Psi_{l^{\prime}}\right)+\beta\left(I_{g}^{\tau}+\eta_{l^{\prime}}^{\tau}\left(\Psi_{l^{\prime}}\right)\right)+ \\
\beta\left(I_{g}^{\tau}-\overline{U^{\prime \tau}\left(K_{a}\right)}\right)^{2}
\end{array}\right)
$$

s.t. constants: (13), (14), (15), (17), (24).

An important challenge to solve this optimisation problem is to know user's temporal behavior as a combined activity of 'Web \& App' i.e. the lack of knowledge of timevarying updates in a user profile. This change in temporal behavior is affected by the profiling derived by app's usage Eq. (13), browsing history/searches Eq. (14), and interactions with ads Eq. (15). This problem would become even more challenging when there is an irregular activity observed by a user e.g. high app's/web usage during weekends etc. Hence, we develop an optimal control algorithm, using Lyapunov optimization, for identifying updates in user profiles as a result of communication requests between the mobile device and the advertising system (analytics) entities, see Section 5. Recall that ad/analytics SDKs enable these requests for tracking and profiling users for their 'Web \& App' activities.

\subsection{Problem relaxation}

To solve the optimisation problem (26), we consider its relax version using (16) to relax constraints (13), (14), and (15). The average expected change in a user profile $I_{g}^{t}$ is given by:

$$
\overline{I_{g}^{t}}=\lim _{t \rightarrow \infty} \frac{1}{t} \sum_{\tau=1}^{t} \mathbb{E}\left\{C_{t}\left(\eta_{l}\left(\Psi_{l}\right)\right)\right\}
$$

From Eq. (16), it is clear that the profile evolves over time e.g. over time slot $t$ and $t+1$ we have $I_{g}^{t}=C_{t+1}\left(\eta_{l}\left(\Psi_{l}\right)\right)+$ 
$C_{t}\left(\eta_{l}\left(\Psi_{l}\right)\right)$. Hence, we take its expected values on both sides of Eq. (16) and equate it to Eq. (27), we have:

$$
\sum_{\tau=1}^{t} \mathbb{E}\left\{C_{t}\left(\eta_{l}\left(\Psi_{l}\right)\right)\right\}=C_{t-1}\left(\eta_{l}\left(\Psi_{l}\right)\right)+C_{t}\left(\eta_{l}\left(\Psi_{l}\right)\right)
$$

Recall that $C_{t}\left(\eta_{l}\left(\Psi_{l}\right)\right)$ is the initial weightages of profiling interests as the profile evolves. Similarly, as mentioned earlier that, these changes are bounded by finite $\min$ and $\max$ bounds i.e.:

$$
C_{t}^{\min }\left(\eta_{l}\left(\Psi_{l}\right)\right) \leq C_{t}\left(\eta_{l}\left(\Psi_{l}\right)\right) \leq C_{t}^{\max }\left(\eta_{l}\left(\Psi_{l}\right)\right)
$$

Divide both sides of Eq. (28) by $t$ and take $t \rightarrow \infty$. Following:

$$
\overline{I_{g}^{t}}=0
$$

Consequently, we have the following relaxed version of objective function:

$$
\min \lim _{t \rightarrow \infty} \sum_{\tau=1}^{t}\left(\begin{array}{l}
C^{\tau} \eta_{l^{\prime}}\left(\Psi_{l^{\prime}}\right)+\beta\left(I_{g}^{\tau}+\eta_{l^{\prime}}^{\tau}\left(\Psi_{l^{\prime}}\right)\right)+ \\
\beta\left(I_{g}^{\tau}-\overline{U^{\prime \tau}\left(K_{a}\right)}\right)^{2}
\end{array}\right)
$$

s.t. constants: (16), (17), (24).

Now the main challenge of solving objective (31) is to minimise the variance of $\overline{U^{t}\left(K_{a}\right)}$ in order to protect user's app's usage behavior i.e. to know all the future usage of apps and hence suggest the (automatic) use of newly suggested obfuscation apps i.e. $\overline{U^{\prime t}\left(K_{a}\right)}$. Similar to Eq. (30), we can also show that $\overline{U^{t}\left(K_{a}\right)}=0$ i.e. to prove that decision variable is independent of $U^{t}\left(K_{a}\right)$. Hence, the above optimisation problem can be solved without the information of $\overline{U^{\prime t}\left(K_{a}\right)}$ at different time slots $t$, as detailed below.

Proof: From Eq. (21), it can be shown that:

$$
\lim _{t \rightarrow \infty} \frac{1}{t} \sum_{\tau=1}^{t} \mathbb{E}\left\{\left(\left(\overline{U^{\prime \tau}\left(K_{a}\right)}\right)^{2}-2 I_{g}^{\tau} \overline{U^{\prime \tau}\left(K_{a}\right)}\right)\right\}
$$

The above equation is independent of the choice of $\overline{U^{\prime \tau}\left(K_{a}\right)}$, hence:

$$
\lim _{t \rightarrow \infty} \frac{1}{t} \sum_{\tau=1}^{t} \mathbb{E}\left\{I_{g}^{\tau}\right\}
$$

As shown in Eq. (20), sum over all $t$, take the expectation of both sides and take $t \rightarrow \infty$, we have the following:

$$
\begin{gathered}
\lim _{t \rightarrow \infty} \frac{1}{t} \sum_{\tau=1}^{t} \mathbb{E}\left\{I_{g}^{\prime} \tau\right\}=\lim _{t \rightarrow \infty} \frac{1}{t} \sum_{\tau=1}^{t} \mathbb{E}\left\{I_{g}^{\tau}\right\}+ \\
\lim _{t \rightarrow \infty} \frac{1}{t} \sum_{\tau=1}^{t} \mathbb{E}\left\{\overline{U^{\prime \tau}\left(K_{a}\right)}\right\} \\
=\overline{I_{g}^{t}}+\overline{U^{\prime} t\left(K_{a}\right)}
\end{gathered}
$$

From Eq. (30), we conclude:

$$
\lim _{t \rightarrow \infty} \frac{1}{t} \sum_{\tau=1}^{t}\left\{I_{g}^{\prime} \tau\right\}=\overline{U^{\prime} t\left(K_{a}\right)}
$$

The relax version of our objective function in Eq. (31) can be re-written as:

$$
\lim _{t \rightarrow \infty} \sum_{\tau=1}^{t}\left(C^{\tau} \eta_{l^{\prime}}\left(\Psi_{l^{\prime}}\right)+\beta\left(I_{g}^{\tau}+\eta_{l^{\prime}}^{\tau}\left(\Psi_{l^{\prime}}\right)\right)+\beta\left(I_{g}^{\tau}\right)\right)
$$

Note that this is an optimised version of (24) i.e. the scenario of introducing medium disruption in a user profile and it achieves balance between user privacy and targeted ads. The other scenarios of low (23) and high (25) profile disruption will have no effect over the (36) except low and high costs and trade-off between privacy and targeted ads.

\section{Optimal Control Algorithm}

We design a control algorithm for identifying communication requests between the mobile device and the analytics server within an advertising ecosystem, to achieve an optimal solution to (26) for identifying time varying updates in user profiles. Let $R_{l}^{t}$ represents an advertising request reported at $t$ via ad/analytics SDK i.e. either ad request for display/ad click in apps/web or web searches/hisoroty; recall that a request may or may not introduce $l$ ( $l$ is profiling interest category introduced during profile updating or evolution) in a user profile $I_{g}^{t}$, alternatively, a change in user profile with an addition of $C_{t}\left(\eta_{l}\left(\Psi_{l}\right)\right)$ during the profile evolution, as described in Section 3.5. Hence:

$$
R_{l}^{t}=I_{g}^{t}-C_{t}^{\min }\left(\eta_{l}\left(\Psi_{l}\right)\right)
$$

Subsequently, the $R_{l}^{t}$ is a shifted version of $I_{g}^{t}$ and can be described as:

$$
R_{l}^{t+1}=I_{g}^{t}+R_{l}^{t}
$$

\subsection{Lyapunov optimisation}

For $n$ requests, we have $R_{l}^{t}=R_{l}^{1}, \cdots, R_{l}^{n}$, following, the quadratic Lyapunov function for each $t$ is given as:

$$
Q\left(R_{l}^{t}\right)=\sum_{\tau=1}^{t}\left(R_{l}^{\tau}\right)^{2}
$$

Similarly, the corresponding Lyapunov drift i.e. drift-pluspenalty, can be defined as:

$$
\Delta R_{l}^{t}=\mathbb{E}\left\{Q\left(R_{l}^{t+1}\right)-Q\left(R_{l}^{t}\right) \mid I_{g}^{t}\right\}
$$

To stabilize the upper bound on Eq. (36), while effective profiling process i.e. the number of interests drawn, to minimise cost of newly suggested apps, and to minimise the variance of $\overline{U^{t}\left(K_{a}\right)}$ for preserving user privacy of apps usage behavior, the control algorithm can be designed to minimise the following drift-plus-penalty on each time slot $t$ :

$$
\Delta R_{l}^{t}+V p(t)
$$


Here, $p(t)$ is the penalty for minimising the upper bound on Eq. (36) i.e. minimise $p(t)=$ $\mathbb{E}\left\{C^{t} \eta_{l^{\prime}}\left(\Psi_{l^{\prime}}\right)+\beta\left(I_{g}^{t}+\eta_{l^{\prime}}^{t}\left(\Psi_{l^{\prime}}\right)\right)+\beta I_{g}^{t}\right\}, V>0$ is a nonnegative parameter chosen as a desired effect of performance of trade-off over the objective function. This approach does not require the knowledge of all future events i.e. the lack of knowledge of time varying updates in a user profile and the apps usage behavior. Eq. (41) can be re-written as:

$$
\Delta R_{l}^{t}+V p(t)
$$

Lemma: The upper drift bound. For the control policy that satisfies the constraints on Eq. (36), we have the following drift-plus-penalty condition holds:

$$
\mathbb{E}\left\{\Delta R_{l}^{t}+V p(t) \mid Q\left(R_{l}^{t}\right)\right\} \leq B+V p^{\prime}(t)-\varepsilon \sum_{\tau=1}^{t}\left(R_{l}^{\tau}\right)
$$

$\varepsilon$ is similar to $\beta$ and can be controlled to achieve the average apps usage time to preserve privacy of user's app usage behavior and to achieve a trade-off between user privacy and targeted ads. $p^{\prime}(t)$ is the desired target for the time average of $p(t)$. $B$ can be defined as:

$$
\begin{aligned}
B & =\frac{1}{2} \max \left\{C_{t}^{\max }\left(\eta_{l}\left(\Psi_{l}\right)\right), C_{t}^{\min }\left(\eta_{l}\left(\Psi_{l}\right)\right)\right\} \\
& \frac{1}{t} \sum_{\tau=1}^{t} \mathbb{E}\{p(\tau)\} \leq p^{\prime}(t)+\frac{B}{V}+\frac{\mathbb{E}\left\{R_{l}^{1}\right\}}{V t}
\end{aligned}
$$

Proof: Taking expectations of both sides of above drift-plus-penalty, we have:

$$
\begin{gathered}
\mathbb{E}\left\{\Delta R_{l}^{t}\right\}+V \mathbb{E}\{p(t)\} \leq B+V p^{\prime}(t)-\varepsilon \sum_{\tau=1}^{t}\left(R_{l}^{\tau}\right) \\
\mathbb{E}\left\{R_{l}^{t}\right\}-\mathbb{E}\left\{R_{l}^{1}\right\}+V \sum_{\tau=1}^{t} \mathbb{E}\{p(\tau)\} \leq \\
\left(B+V p^{\prime}(t)\right) t-\varepsilon \sum_{\tau=0}^{t} \mathbb{E}\left\{Q\left(R_{l}^{\tau}\right)\right\}-\mathbb{E}\left\{R_{l}^{1}\right\} \\
+V \sum_{\tau=0}^{t} \mathbb{E}\{p(\tau)\} \leq\left(B+V p^{\prime}(t)\right) t \\
V \sum_{\tau=1}^{t} \mathbb{E}\{p(\tau)\} \leq p^{\prime}(t) V t+B t+\mathbb{E}\left\{R_{l}^{1}\right\}
\end{gathered}
$$

Dividing by $V t$ and rearranging terms proves the bound on average penalty, concludes the proof.

\subsection{Control algorithm}

The main objective of the control algorithm is to minimise the drift-plus-penalty bound subject to the constraints of (36) at each time slot $t$. This control algorithm optimally selects the minimum and maximum bounds over the selection of obfuscation apps, the rate at which these apps needs to run according to the variation in $R_{l}^{t}$ and by observing the current states of $I_{g}^{t}$ i.e. a solution to the following optimisation problem:

$$
\begin{aligned}
& \min _{l}^{t}+V\left(C^{t} \eta_{l^{\prime}}\left(\Psi_{l^{\prime}}\right)+\beta\left(I_{g}^{t}+\eta_{l^{\prime}}^{t}\left(\Psi_{l^{\prime}}\right)\right)+\beta I_{g}^{t}\right) \\
& \text { s.t. } \\
& C_{t}^{\min }\left(\eta_{l}\left(\Psi_{l}\right)\right) \leq C_{t}\left(\eta_{l}\left(\Psi_{l}\right)\right) \leq C_{t}^{\max }\left(\eta_{l}\left(\Psi_{l}\right)\right) \\
& R_{l}^{t}=I_{g}^{t}-C_{t}^{\min }\left(\eta_{l}\left(\Psi_{l}\right)\right) \\
& I_{g}^{t}=\sum_{\forall t} C_{t}\left(\eta_{l}\left(\Psi_{l}\right)\right) \forall l \in\{[1, \psi], m, n\}
\end{aligned}
$$

The performance of this algorithm is to achieve minimised objective during when changes occur in profiling process at $t$ i.e. the stable and during the profile development and profile evolution processes, in order to solve (48) as a mixed integer non-linear programming optimisation problem. The following cases:

Stable state: Recall that during this state, no changes occur in the profiling process i.e. $C_{t}\left(\eta_{l}\left(\Psi_{l}\right)\right)=0$. Hence, the optimal value of (48) is evaluated to:

$$
V\left(C^{t} \eta_{l^{\prime}}\left(\Psi_{l^{\prime}}\right)+\beta \eta_{l^{\prime}}^{t}\left(\Psi_{l^{\prime}}\right)\right)
$$

Let $p\left(R_{l}^{t}\right)$ tracks the advertising requests reported at $t$ via ad/analytics SDK during slot $t$, which is considered minimum i.e. $p^{\min }\left(R_{l}^{t}\right)$, during the stable state, hence, the $\eta_{l^{\prime}}\left(\Psi_{l^{\prime}}\right)$ is selected as $\eta_{l^{\prime}}\left(\Psi_{l^{\prime}}\right)=\min \left\{0, \eta_{l}^{\min }\left(\Psi_{l}\right)\right\}$.

Profile development/evolution state: The profiling process speeds up during this state as a result of high interaction with the analytics servers. Let $p^{\min }\left(R_{l}^{t}\right), p^{\text {avg }}\left(R_{l}^{t}\right)$, and $p^{\max }\left(R_{l}^{t}\right)$ respectively represents the minimum, average, and maximum; following we present various scenarios for calculating optimal value of (48):

- $\quad$ For $p^{\min }\left(R_{l}^{t}\right)$ :

$$
\text { - } \eta_{l^{\prime}}\left(\Psi_{l^{\prime}}\right)=\max \left\{0, \eta_{l}^{\min }\left(\Psi_{l}\right)\right\}
$$

- $\quad$ For $p^{a v g}\left(R_{l}^{t}\right)$ :

$$
\text { - } \quad \eta_{l^{\prime}}\left(\Psi_{l^{\prime}}\right)=\min \left\{\eta_{l}^{\min }\left(\Psi_{l}\right), \eta_{l}^{\max }\left(\Psi_{l}\right)\right\}
$$

- $\quad$ For $p^{\max }\left(R_{l}^{t}\right)$ :

$$
\text { - } \eta_{l^{\prime}}\left(\Psi_{l^{\prime}}\right)=\max \left\{\eta_{l}^{\max }\left(\Psi_{l}\right), \eta_{l}^{\max }\left(\Psi_{l}\right)+\eta_{l^{\prime}}^{\max }\left(\Psi_{l^{\prime}}\right)\right\}
$$

Given these values, the optimal objective can be calculated as:

$$
p\left(R_{l}^{t}\right)+V\left(C^{t} \eta_{l^{\prime}}\left(\Psi_{l^{\prime}}\right)+\beta\left(I_{g}^{t}+\eta_{l^{\prime}}^{t}\left(\Psi_{l^{\prime}}\right)\right)+\beta I_{g}^{t}\right)
$$

The lower values on (49) and (50) and the corresponding control values are calculated to evaluate the optimal values of our objective. 


\section{Performance Analysis}

We now analyse the feasibility and performance of the proposed model using various evaluation metrics, in addition to, we discuss the POC implementation of our proposed model. The applicability of the proposed system is discussed in Section 8.3.

\subsection{Evaluation metric}

We define utility and further elaborate the cost of newly selected apps to provide insights on usability of the newly selected apps usage. The authors in [17] describe utility as the success rate for removal of private query tags i.e. the magnitude suppression of original user preferences.

\subsubsection{Utility}

Let $D\left(\eta_{p}\left(\Psi_{p}\right)\right)$ is the dominating private interest category. We define utility based on two components: first, from the effectiveness of privacy protection, we use as metric the level of reduction $R_{p}$ of $\eta_{p}\left(\Psi_{p}\right)$ of a selected private category $\Psi_{p}$ in an Interest profile, achieved via newly suggested obfuscating apps, with $\frac{D\left(\eta_{p}\left(\Psi_{p}\right)\right)}{D\left(\eta_{l}\left(\Psi_{l}\right)\right)}$. Here $D\left(\eta_{l}\left(\Psi_{l}\right)\right)$ is the new dominating ratio resulting from using apps in $S_{o}\left(S_{o}\right.$ is the new set of apps other than $S_{a}$; the selection of these apps has been detailed in Section 4.2).

Secondly, we introduce usability $U_{s}$ of the selected obfuscating apps that relates to the probability that a user would actually utilise these apps, rather than just install and run it for privacy protection. The $U_{s}$ of an app $a_{o} \in S_{o}$, in regards to a user with a specific Context profile, as the ratio of similarity between this app and any of the apps in $S_{a}$ and the maximum similarity of any other app from $\mathcal{A}$, not present in $S_{a}$ and apps from the same set:

$$
\begin{aligned}
& U_{s}=\min \left(\frac{\operatorname{sim}\left(a_{o}, a_{i, p}\right)}{\operatorname{sim}\left(a_{q, r}, a_{i, p}\right)}\right): \\
& a_{i, p} \in S_{a}, a_{q, r} \notin S_{a}
\end{aligned}
$$

Combining the two, the total utility can then be calculated as: $U_{T}=R_{p}+U_{s}$.

\subsubsection{Cost and resource overhead}

The cost and resource overhead, can be considered as equivalent terms in the context of introducing new activities, which result in usage time and other resources e.g. battery usage, however, for the sake of clarity we use two separate terms. As mentioned earlier, cost $C$ is termed as a metric that relates to the reduction of overall usage time available (by use of obfuscating apps) to original (non-obfuscating) apps.

In the basic scenario where, the average introduction of newly selected apps, the usage of all apps is uniformly distributed within a time period in correspondence to $U^{t}\left(K_{a}\right)$; this is equivalent to the ratio of the number of obfuscating apps in $S_{o}$ (that need to be installed and used to protect privacy) and the size of original apps set $S_{a}$. Cost is therefore defined as:

$$
C=\left|S_{o}\right| /\left|S_{a}\right|
$$

We consider the resource overhead $R$, to be the overall resource usage by running the newly suggested apps. Hence, there will be a corresponding overhead $R_{i, j}^{t}$ for each app $a_{i, j}$ at time slot $t$, comprising broadly of communication $R_{c}^{t}\left(a_{i, j}\right)$, processing $R_{p}^{t}\left(a_{i, j}\right)$, and battery consumption overheads $R_{b}^{t}\left(a_{i, j}\right)$.

$$
R_{i, j}^{t}=R_{c}^{t}\left(a_{i, j}\right)+R_{p}^{t}\left(a_{i, j}\right)+R_{b}^{t}\left(a_{i, j}\right)
$$

Following, we present further detail on resource usage and then experimentally evaluate various components (see Section 7.5) of the overall resource overhead $R$.

\subsection{Evaluating resource use}

We rely on various utilities of Android $S_{D K}{ }^{16}$ to automate various measurements for processing and battery consumption. As an example, we execute $\mathrm{adb}$ shell top $-\mathrm{m} 10$ within the Process $\mathrm{p}=$ Runtime.getRuntime ().exec ("command") to determine $C P U$ processing of each running app. Similarly, to evaluate battery's current status, we use adb shell dumpsys battery | grep level command by initiating the startActivity() of the Intent utility of Android SDK. To measure this, we first charge the battery to $100 \%$ and then run the app for one hour, while connected to WiFi network. We envisage that running of newly suggested apps would be used over WiFi to reduce communication costs (specifically for users with limited mobile network packages), although, in real life scenario, users are likely to utilise apps (equally) on a mobile network that would results different magnitudes of resource overheads.

Similarly, we utilise the traffic captured during our experiments in order to evaluate communication overhead; described in Section 7.2.

In addition to above, we evaluate another resource usage overhead i.e. storage space consumption. This can be further classified into installation storage space, cache storage i.e. temporary stored data, e.g. cookies stored on phone, internal data size i.e. storage used for apps' files, accounts, etc., which are respectively calculated using codeSize, cacheSize, and dataSize of PackageStats package of Android SDK. Note that we automated all these through an app, and all the experimentation discussed in next section, using Android Debug Bridge ${ }^{17}$ of Android SDK that enables communication between a PC and connected Android devices.

\section{Performance Evaluation}

We now discuss details of various components of 'System App' implementation and experimental evaluation and further provide insights on resource overheads.

\subsection{System App: The obfuscating system}

We have implemented a POC 'System App' of our proposed framework. Various components are presented on left side of Figure 6 i.e. 'User Environment', which introduces changes to the user side with a range of different functionalities e.g. it implements local user profiling both for Context profile and Interests profiles (as detailed in Section 4.2), protects user's privacy for their (private) sensitive attributes,

16. https://developer.android.com/studio

17. https://developer.android.com/studio/command-line/adb 
and preserves user privacy for their apps usage behavior (Section 4.3). In addition, it implements our proposed online control algorithm for jointly optimising user privacy and cost; presented in Section 5. To enable this functionality, a user need to install and run the 'System App'; this approach is similar to existing app recommender systems, e.g. AppBrain $^{18}$. The 'System App' acquires information about the set of currently installed apps on a device, interacts with user in regards to selection of private attributes to be protected. Furthermore, it evaluates the list of candidate obfuscating apps and presents to the user and automates the process of installation and runing of these apps.

The 'System App' sends the installed app information to the 'System Engine' for calculating obfuscating apps and automates the installation and running of these apps, as shown in Figure 6. We have used various utilities of the Android SDK in our implementation. E.g., PackageManager ${ }^{19}$ is used to retrieve the list of installed apps. The meta data of the installed apps, such as app name, permissions etc., are obtained by calling the function getInstalledApplications (PackageManager.GET_META_DATA). The 'System En-

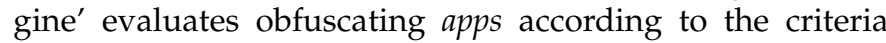
discussed in Section 4.2 by examining the 'Local app repository'; we suggest that this repository is updated by the advertising system so that the 'System App' can calculate obfuscating apps from various app's categories.

The 'System Engine' module forwards the list of obfuscating apps to the 'System App'. Each app is displayed to the user as an accessible hyperlink to Google Play (or 'Apple's App Store') store, which is done by invoking the startActivity (new Intent (Intent.ACTION_VIEW, Uri.parse ("market://details?id=" +appPackageName))).

The activity name (the app name that can be recognised by app market of the app to be installed) is specified using appPackagename function using the Intent class. These apps are automated and run using the startActivity utility of Android SDK for a specified amount of time that is required to generate new profile interests in the ad system.

\subsection{Experimental setup}

In this work, we mainly focus on Google $\mathrm{AdMob}^{20}$ since it is the leading marketplace in mobile user profiling and has captured the online digital advertising market, however, we note that the proposed methods can be effortlessly applied to other ad/analytic networks. We carry out various experimentations for preserving profiling privacy (i.e. $\left.I_{g}^{t}+\eta_{l^{\prime}}^{t}\left(\Psi_{l^{\prime}}\right)\right)$, apps usage $\left(I_{g}^{t}-U^{\prime t}\left(K_{a}\right)\right)$ and evaluating various resource overheads $R$. To evaluate this, we select apps from 27 random (sample) categories; we note that Google Play store has organised apps into 37 (considering 'Games' as a single category) various categories e.g. 'Entertainment', 'Lifestyle' etc. For these experimentations, we select top 100 free apps from randomly chosen 27 categories and further narrow down it to 10 highest ranked apps from

18. https:/ /www.appbrain.com/

19. https://developer.android.com/

20. https://apps.admob.com each category. As mentioned earlier that the Google user profile is partly based on mobile apps usage and relevance of received ads, hence, we had to ensure that the tested apps receive ads.

We set a second (mapping) experimental setup; we select one mobile $a p p$ from the top list of any category and run it for a period of up to 96 hours; note that this process was automated, as described above in Section 7.1. The purpose of these experiments was to evaluate mapping of specific Context profile to Interest profile i.e. $K_{a} \rightarrow I_{g}$, as discussed in Section 3.2. This helps in determining contribution of individual app in an Interest profile. We note that findings from these experiments help in selecting newly selected apps for various disturbances in user profiles i.e. achieving different trade-offs between privacy and targeted ads, as explained in Eq. (23), (25), and (24) e.g. $0<\eta_{l^{\prime}}\left(\Psi_{l^{\prime}}\right) \leq \eta_{l}^{\min }\left(\Psi_{l}\right)$ for lower privacy and higher targeted ads. These experiments have taken around 3 months to complete for the 270 highest ranked apps from 27 random apps categories.

The ads traffic, including the control traffic exchanged for tracking/profiling purposes, was collected using tcpdump ${ }^{21}$, cleansed and saved to a local database during entire experimentations. We reset the profile ${ }^{22}$ before starting each experiment in order to make sure that the Interest profile is only resulting from the currently installed and actively used apps. In addition, we set up a phone with the same configuration however with 'Opt-out of Ads Personalisation' enabled in Google Settings system app. The purpose of this phone is to have a base reference for both newly generated user profile and received targeted ads. These experiments were run for all the selected categories for 24/7 for 5 months; due to practical limitations of these experiments, we only use 10 smartphones in parallel.

We also used the collected traffic from these experiments for calculating the resource usage; detailed in Section 6.2.

\subsection{Trade-off between apps usage privacy and cost of profile disruption}

Figure 8 compares the apps usage profile for lower and higher profile disruption by introducing lower (as shown in (a)) and higher (i.e. (b)) activity of newly suggested apps; note the original user profile activity is shown with rectangular boxes; already discussed in Figure 4. We first divide the time into $5 \mathrm{~min}$ duration bins and then record the number of ads requests in each bin (there are different number of ad requests by each app; as detailed in Section 7.5); each dot (in plot) in both Figures 8 (a) and (b) show the ad request frequency during the 24 hours time (shown on $\mathrm{x}$-axis) and their corresponding ads requests frequency (i.e. shown on y-axis).

The newly suggested apps were run during various (day/night) times where there was no original $\left(K_{a}\right) a p p^{\prime} \mathrm{s}$ activity e.g., during 1am-6am, as indicated in both figures. We note that, during 1am-6am with 'lower app usage and low profile disruption', newly apps request 27 ad (binned)

21. www.tcpdump.org

22. The profile was reset by using 'Reset advertising ID' option in Google Settings system app i.e. Google Settings $\rightarrow$ Ads $\rightarrow$ Reset advertising ID. 
requests with a total of fetching 93 ads, while it is respectively 93 and 1323 for 'higher app usage and high profile disruption'. In addition, the average number of ad requests and corresponding ads fetched were 19.6 and 60 for 'lower app usage and low profile disruption', while it was 77.6 and 809.6 for 'higher app usage and high profile disruption'. Overall, there were 340 and 5068 ad requests respectively with two options of profile disruptions; note that this also has effect over various overheads, in particular, the Communication overhead, also discussed later in Section 7.5.

\subsection{Privacy protection vs. ads distribution}

Figure 9 (a) and (b) show frequency distribution of ads for lower and higher apps usage; the percentage of ads for presented ads in different time-bins is also shown on top of each bar. For each time bin, the presented ads were ranged from 1 to 16 ads requests, in particular, the frequency of these requests range from 1 to 9 ads requests for 'lower apps usage', which also attracted lower number of ads i.e. only 85 ads to completely apps usage privacy as shown in Figure 9 (a). Similarly, for 'higher apps usage', the ads requests range from 1 to 16 per time bin with an average of 32 ads for all frequency of ads, with a total of 480 ads in order to completely preserve user's privacy. Recall that, the selection of such apps usages are mainly done for two purposes i.e. to protect apps usage privacy and to affect the targeted ads.

\subsection{Privacy protection vs. resource use}

It is reported by previous works [4], [18], and is also evident in our experimental results, that the ads (and its related tracking traffic) are the major contributor to the communication $R_{c}^{t}\left(a_{i, j}\right)$ overhead. Hence, we approximate the communication cost by the traffic generated by ads and their related actions by users.

We note that, from the bandwidth viewpoint, the ads traffic is characterised by various components: The ad refresh rate $^{23}$ (technically it is the inter-arrival time of two consecutive ads); their correspondence with various ad/analytic servers with an advertising ecosystem; contacts with CDN for downloading various ad components e.g. images etc.; the number of objects associated with an ad along with their sizes; and communication with various servers during interactions with an ad. Table 2 shows various ad related objects and control messages, along with their sizes; determined from collected traffic traces.

During our experimentations, we examined, from collected traces, that an ad size is $16 \pm 4 \mathrm{KBs}$, which (on average) contains $8-10$ objects (e.g. JavaScript files, images etc.) with an average of 30-35 request/response messages. In addition, we note that ad refresh rates vary between 20-60 seconds ${ }^{24}$, with distinct values of 20,30, 45 and 60 seconds, which respectively to $36 \%, 47 \%, 15 \%$ and $2 \%$ of all the tested apps. Since the ad sizes do not vary widely, hence the communication overhead for introducing lower disturbance

23. AdMob refers this to rate, which is deterministic for every app and is configured by apps developer at the time of registering apps on Google Play store.

24 . We note that supported values are 12-120 seconds in Google AdMob. in a user profile can be further minimised by selecting those apps that have maximum overall ad refresh rate; note that this information is already available with any ad network e.g. Google AdMob.

TABLE 2: The ad-related objects and control messages along with their average size in bytes.

\begin{tabular}{|l|c|l|c|}
\hline Message Type & Size (bytes) & Message Type & Size (bytes) \\
\hline 200 Ok & 496 & POST & 350 \\
\hline DNS Query Request & 68 & Ok/(PNG) & 1300 \\
\hline GET /pagead/images & 578 & Ok/(GIF) & 1000 \\
\hline DNS Query Response & 334 & Ok/(JPEG) & 1300 \\
\hline Ok/(text/html) & 1200 & TCP reassembled PDU & 1434 \\
\hline GET /geocode & 224 & no content & 396 \\
\hline GET /simgad & 252 & Ok/(application/json) & 240 \\
\hline GET /mads/gma & 685 & Ok/(text/javascript) & 800 \\
\hline GET /imp & 244 & Ok/(text/css) & 824 \\
\hline GET /generate_204 & 244 & TCP Ack & 66 \\
\hline GET /csi & 595 & TCP Syn & 74 \\
\hline
\end{tabular}

Figure 10 shows the distribution of bandwidth used by apps during our experimentations per our experimental setup, shown in Section 7.2. The proportion of apps that have consumed the respective bandwidths are also shown on top of each bar e.g. $26.30 \%$ of the apps (which corresponds to 71 apps) consumes a high bandwidth of 4.0-4.5MB. In addition, we note that apps that frequently fetch ads i.e. apps with ad refresh rates of 20 and 30 seconds, utilise an average bandwidth of $3.0-5.5 \mathrm{MB}$; these apps represent around $70 \%$ of all the experimented apps. Note that the selection of such apps can be used for introducing higher disruption in user profiles, to attract lower targeted ads, and to achieve high apps usage privacy. The remaining $30 \%$ of the apps (i.e. apps with ad refresh rates of 45 and 60 seconds) utilise between 0.5-2.5MB communication bandwidth.

Subsequently, we evaluate the processing overhead introduced by experimented apps. We note that the measured CPU usage varies, although not widely, across different apps: the CPU-intensive apps such as those from the 'Games' category use between $25 \%$ to $30 \%$ of the CPU power; lessinteractive apps such as Notes use between $15 \%$ to $20 \%$.

Similarly, the battery consumption measured in our experiments shows a relatively low variations between various apps, with between $30 \%$ to $40 \%$ of the total battery (i.e. 100\%) being used by each app during the measurement period.

In addition to above basic overheads, we evaluated storage space consumed by newly suggested apps; we mainly determined the installation, data, cache storage spaces. These storage spaces vary for apps according to their requirements e.g. a 'language translation' app might do offline text translation, hence it would require to save library files in data storage space quota requiring more space compared to installation storage space. In contrast, the Facebook would consume more data storage space to store user accounts data, search history, group settings, user timeline data etc. In addition, a Google Maps would take more cache storage space to save user's searched places history etc. Table 3 presents few representative apps for combination of these storage space requirements; following we present further details.

Figures 11(a) through (c) show the distribution of storage spaces of the experimented apps; the proportion of apps that requires respective storage space are also shown on top of each bar. It can be observed, for installation storage, 


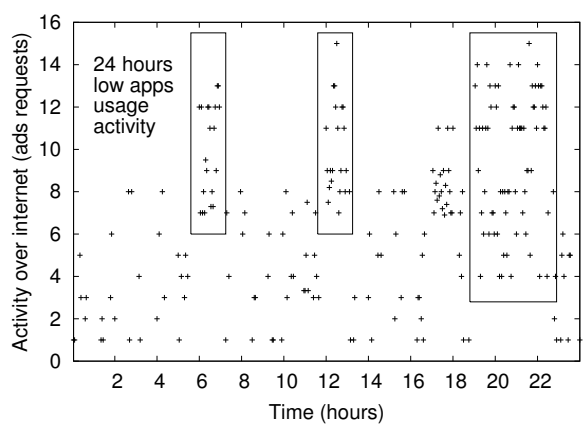

(a) Lower profile disruption

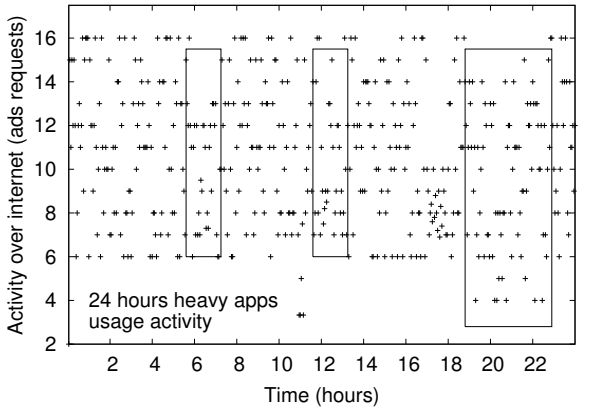

(b) Higher profile disruption

Fig. 8: Lower activity of newly suggested apps for lower (a) and higher (b) profile disruption.

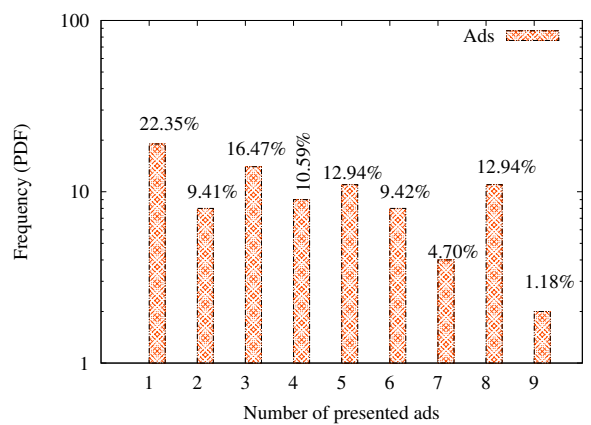

(a) Lower apps usage and lower ads distribution

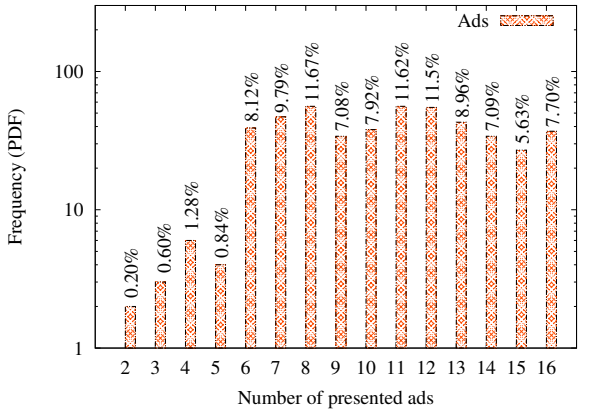

(b) Higher apps usage and higher ads distribution

Fig. 9: Distribution of ads by lower (a) and higher (b) apps usage.

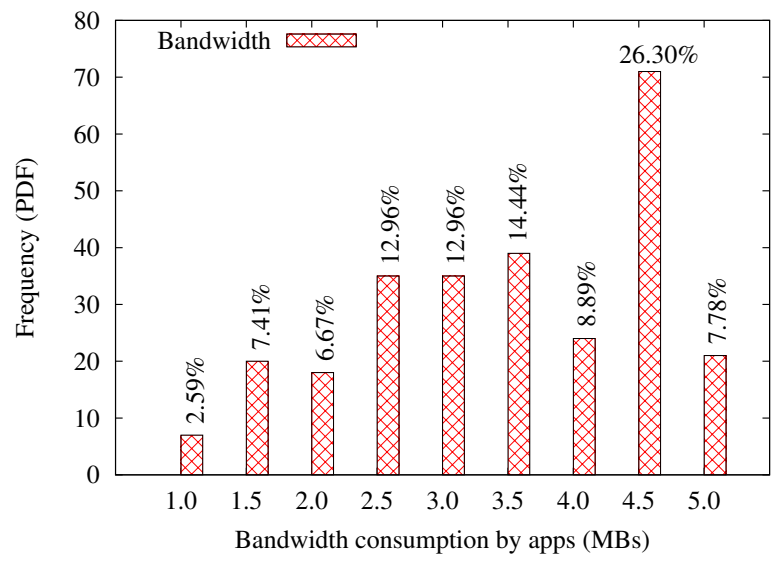

Fig. 10: Bandwidth consumption (MB) calculated for ads by tested apps during experimentations.

that nearly half $(54 \%)$ of the experimented apps acquires lower storage space i.e. $0.5 \mathrm{MB}$ to $10 \mathrm{MB}$, while only $1.48 \%$ of these apps consume relatively higher storage space of 50 60MB. Similar observations can be found for data and cache overhead, as shown in Figures 11 (b) and (c). In conclusion, we observe that vast majority of these apps use a lower amount of storage space; e.g., $80 \%, 97 \%$, and $98 \%$ of all apps belong to the lowest storage space bins, i.e. within the range of $0.5-20 \mathrm{MB}, 0.01-20 \mathrm{MB}$, and $0.01-10 \mathrm{MB}$ storage
TABLE 3: Representative apps with various combination of storage space requirements.

\begin{tabular}{|l|c|c|c|}
\hline \multirow{2}{*}{ App Names } & \multicolumn{3}{|c|}{ Storage space consumed } \\
\cline { 2 - 4 } & Installation & Data & Cache \\
\hline Youtube & 117 & 2.57 & 361 \\
\hline Subway Surf & 156 & 85.98 & 15.57 \\
\hline Chrome & 86.88 & 15.46 & 1.53 \\
\hline File Manager & 8.37 & 28.04 & 17.83 \\
\hline Foxit PDF & 96.42 & 1.17 & 32.91 \\
\hline London City Guide & 60.98 & 88.12 & 4.56 \\
\hline Google Play Store & 93.61 & 7.48 & 22.25 \\
\hline Skype & 62.95 & 28.22 & 13.41 \\
\hline Babel & 51.98 & 14.79 & 0.02 \\
\hline English For Kids & 16.45 & 3.55 & 10.81 \\
\hline Viber & 159 & 17.35 & 0.12 \\
\hline Google Translate & 5.53 & 392 & 2.04 \\
\hline Adobe Acrobat & 20.91 & 0.47 & 0.22 \\
\hline Amazon Kindle & 53.30 & 123.33 & 4.98 \\
\hline TripView Lite & 28.21 & 5.92 & 2.09 \\
\hline Gmail & 74.24 & 22.28 & 0.34 \\
\hline
\end{tabular}

consumption, respectively for installation, data, and cache storage overheads.

\section{Discussion}

We discuss the applicability of our framework in current advertising ecosystem, in addition to, comparing the proposed framework for various privacy protection approaches for the presented threat model. 


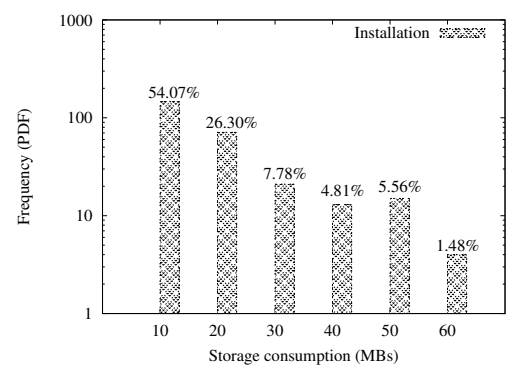

(a) Installation Storage

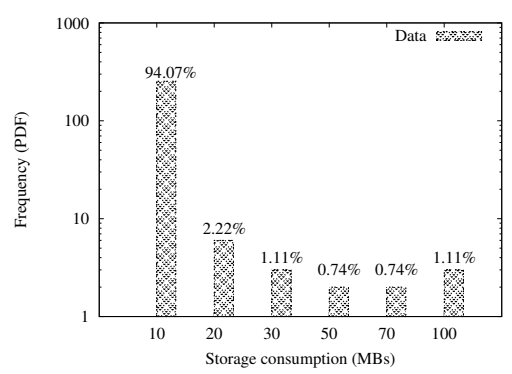

(b) Data Storage

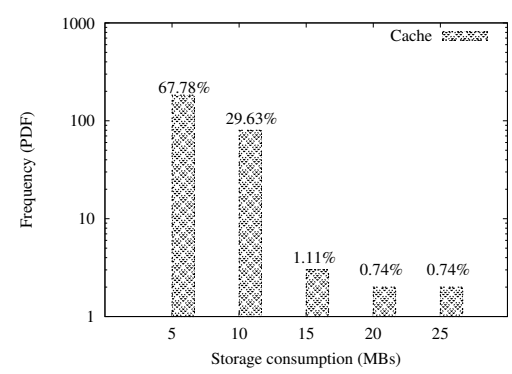

(c) Cache Storage

Fig. 11: Storage space consumed by experimented apps for (a) Installation (b) Data and (c) Cache.

\subsection{Protecting sensitive profiling interests via Differen- tial privacy}

The concept of differential privacy ${ }^{25}$ was introduced in [19], a mathematical definition for privacy loss associated with any released data fetched from a database. A deeper understanding of differential privacy and its algorithms can be found in [20]. Let $D_{1}=g_{k, l} \in S$ i.e. the set of interests in a user profile, and $D_{2}=g_{k, l}^{\prime} \in S_{g}$, where $\Psi_{l} \neq \Psi_{l}^{\prime}$ and $g_{k, l}^{\prime}$ is the profiling interest(s) other than the primary set of interests defined by an advertising company, $\Psi_{l}$ is a selected private interests category that the user wants to protect. Subsequently, the randomised function $K$ gives differential privacy for these two data sets as:

$$
\mathrm{P}_{r}\left[K\left(D_{1}\right) \in S\right] \leq \exp (\varepsilon) \times \mathrm{P}_{r}\left[K\left(D_{2}\right) \in S\right]
$$

We examine the use of other privacy protection mechanisms that can also be utilised e.g. private information retrieval, anonymisation, randomisation, Blockchain-based solutions, however, we note that these solutions do not fully protect user's privacy for the presented threat model; following we provide a comparative analysis of use of various other privacy protection mechanisms.

\subsection{Comparison with other privacy protection ap- proaches}

Table 4 compares various privacy protection mechanisms using different parameters, evaluated in our proposed framework. It can be observed that only the proposed mechanism of introducing newly suggested obfuscation apps protects user's privacy for app's usage behavior, since the user has to run these apps in order to protect usage behavior at different periods of the day/night. Similarly, an important parameter is the trade-off between privacy and targeted ads, which can only be achieved using the proposed mechanism and the randomisation. Furthermore, another parameter is to protect user privacy in terms of serving targeted ads (an indirect privacy attack to expose user privacy), which can also be adjusted according to user's needs i.e. 'low-relevant vs. high-relevant interest-based ads'.

25. A C ++ implementation of differential privacy library can be found at https://github.com/google/differential-privacy.

\subsection{Applicability of proposed framework in advertising system}

Overall system functionality: The motivation for protecting user privacy is very much dependent on the way consumers use mobile apps and access internet; users are ever more concerned about preserving their privacy due to an enormous increase in its awareness. Such an example awareness motivation is by exposure of mass surveillance activities ${ }^{26}$ and by unauthorised leaks of personal data. Hence, users have ever more interested in use of personal (bespoke) privacy tools. Thus the proposed framework is considered, inline with the current apps recommender and personalisation systems, that not only suggest usable newly recommended apps but also enable an optimised and cost effective privacy protection mechanisms. As we have seen in Section 7.3, this framework optimises the cost by selecting various number of apps for protecting apps usage privacy, protects user's private interest profile and achieves optimal trade-off between privacy protect and the targeted ads. Furthermore, this framework ensures that the newly recommended apps have an overall good usability to users, based on similarity metric discussed in Section 6.1.

An important user's concern is the issue related to resource use, we note during our mapping experimentation (as detailed in Section 7.2) that these experiments can help to significantly reduce the resource use by selecting appropriate apps that could (subject to availability of such mapping information) effectively preserve user privacy with least overhead. Using these experiments, on the average, the communication cost is around $3 \mathrm{MB}$ (for lower profile disruption) compared to $17 \mathrm{MB}$ for higher profile disruption, to achieve apps usage and profiling privacy. This would motivate users to use such strategy, in particular, those who might be on fixed mobile data plan. As discussed earlier that AdMob profiling is based on 'Web \& App' activity, hence such (mapping) information can also be added for user's web searches/histories; although this app-based strategy is still applicable to protect user privacy for interest profiling as profiling is now done via both 'Web \& App' activity. We envisage that such information could be made derived by approximating user profile based on various related information available, such as search/history keywords, similar to interest mapping discussed in Section 3.2 for $K_{a}$.

26. http://www.theguardian.com/world/the-nsa-files 


\begin{tabular}{|l|c|c|c|c|c|}
\hline Parameters & Proposed & $\begin{array}{c}\text { Differential } \\
\text { Privacy }\end{array}$ & $\begin{array}{c}\text { Cryptographic } \\
\text { mechanisms }\end{array}$ & Randomisation & $\begin{array}{c}\text { Blockchain-based } \\
\text { solutions }\end{array}$ \\
\hline $\begin{array}{l}\text { Apps usage behaviour } \\
\text { privacy }\end{array}$ & Guaranteed & No guarantee & No guarantee & No guarantee & No guarantee \\
\hline Cost $C_{t}$ & Yes & Yes & Yes & Yes & Yes \\
\hline Targeted ads & $\begin{array}{c}\text { Minor to no relevant } \\
\text { ads (adjustable) }\end{array}$ & Yes & Yes & $\begin{array}{c}\text { Minor to no relevant } \\
\text { ads (adjustable) }\end{array}$ & Yes \\
\hline $\begin{array}{l}\text { Trade-off b/w privacy and } \\
\text { targeted ads }\end{array}$ & Yes & No & No & Yes & No \\
\hline Profiling privacy & Yes & Yes & Yes & Yes & Yes \\
\hline
\end{tabular}

TABLE 4: Comparison of proposed framework with other privacy protection mechanisms for various performance parameters.

Server side modifications: Integrating the proposed framework within the existing advertising ecosystem would be fairly straightforward and would only require upgrading tracking and analytics mechanism of the server side i.e. we suggest the advertising system to transfer such functionalities to the client side; where the client remains honest. These changes are mainly related to Aggregation and Analytics servers; these servers would respectively receive the constructed user profile (along with anonymous apps usage statistics without including user's Advertising ID) and other other statistics, e.g. ad impression/clicks etc., required by both advertising system and apps developers.

Client side modifications: A major change on the client side would require implementation via 'System App' i.e. user profiling, optimising user's privacy for targeted ads, their interactions with ads and app usage privacy including the 'Obfuscation engine' for selection of newly suggested apps. Currently, the mobile integrates with the advertising ecosystem via SDK, hence it will mainly require modifications in client's AdMob SDK.

Other privacy attacks: The proposed system protects users privacy against legitimate user profiling and the traffic monitoring/analysis and network surveillance both form the host advertising systems and third-party tracker/analytics. However, we did not address locationbased privacy for ads targeting in much more detail, although this can also be handled by our privacy protection approach since the location is also added as one of the interest in user profile as part of demographics, as detailed in Section 2.1. For location specific ads, a low resolution GPS coordinates can be included in user profile to accommodate advertisers and businesses wishing to advertise to passing trade. In addition, location can be protected using other other protection mechanisms such as 'Tor'; to use in conjunction with our system to prevent such threats.

\section{Related Work}

In our recent work [1] we provide detailed survey on privacy leakages in profiling process, leakage of personal information by advertising platforms and ad/analytics networks, the measurement analysis of targeted advertising based on user's interests and profiling context, in addition to, detailed discussion over ads delivery process in both in-app and in-browser targeted ads. We provide detailed insights on how private information is extracted and exchanged among various advertising entities e.g. between ad/analytics libraries and analytics server in an ad system, similarly, associated privacy threats with third-party tracking, re-identification of private information and detailed overview of data and tracking sharing technologies. In addition, we compare various privacy preserving advertising systems for various capabilities, such as, the underlying architecture used, the privacy mechanisms and the deployment scenarios.

Privacy threats in targeted advertising have been extensively studied in literature e.g., direct and indirect (inferred) leakage of private information [3], [21], [22], [23] or third party ad tracking and visiting [24], [25], [26], [27]. These works show the prevalence of user (from their online data) tracking on both web and mobile environments and demonstrate the possibility of inferring user's PII, such as email addresses, age, gender, relationship status, etc. The ad/analytics libraries (embedded with mobile apps) leak user's personal information to ad system and (is more likely to) third party tracking, which are systematically collected by such libraries. Such example works [28], [29] study information collected by analytics libraries integrated within mobile apps and leaking of private information. Similarly, the authors in [30] show privacy and security risks by analysing 100,000 Android apps and find that majority of the embedded libraries collect and share private information. Other studies [31], [32] find that majority of the mobile apps do not implement private APIs and send private information to ad servers.

Recall that user profiling and ads targeting is carried out based on user's activity over apps and internet; the authors in [33] manually create user profiles and examine that majority of targeted ads were based on the fabricated profiles. In our previous work [14], and later supported by another study [34], we examine various tracking information sent to the ad networks and the level of ads targeting based on such profiling information. Another work [23] investigates the information collected by ad networks using installed apps and reverse engineer the targeted ads. In line with above works, we also study [12] leakage of private sensitive information by examining actual network traffic through vulnerabilities in mobile analytics services, reconstruct the exact user profile of a number of participating volunteers, and further study its influence over ads targeting. This study was mainly for Google Mobile Analytics and Flurry Analytics. Third-party tracking also actively collect, manage and distribute user's sensitive information, which has been widely studied in literature e.g. [26], [35], [36], [37], [38], [39] study the distribution of third-party trackers across web and Android apps and their impact on user privacy. 
Other works [4], [15], [16], [40], [41], [42], [43], suggest the app-based user profiling, stored locally on mobile device. There are various in-app privacy-preserving mobile advertising and personalisation proposals, such as, Adnostic [44], Privad [45], RePriv [46], MobiAd [41], Splitx [47], ProfileGuard [4], [16]. The ProfileGuard that is an app-based profile obfuscation mechanism for protecting user privacy using various obfuscation strategies. Similarly, there are some other solutions that are crypto-based implementation of various techniques under Private Information Retrieval (PIR) [40] and Blockchain-based solutions [15], [48] for decentralised advertising system that enables private profiling and targeted ads.

This work, as opposed to our previous work [4] where it only protects user privacy by reducing dominance level of particular interests, jointly optimises user's profiling privacy, the user's apps usage behavior, and cost of achieving the level of privacy. We provide an online control algorithm that provides a trade-off by achieving between user privacy and targeted ads. This was achieved by optimally identifying updates in user profiles and user's apps usage behavior of actual apps by capturing temporal changes during the profiling process. We mainly address the attacks regarding app-based profiling, ads-based inferencing, and analysing user's behavior by sniffing network traffic of legitimate users.

\section{Conclusion}

The online mobile targeted advertising is growing in popularity, which at the same time has raise serious privacy concerns among individuals, enabled via the use of excellent user-tracking tools. This paper presents an optimal privacy preserving and cost-effective framework for preserving user privacy due to user profiling, ads-based inferencing and ads targeting and (in general) user's behavior over mobile devices. We present a dynamic optimisation framework by first examining the underlying advertising ecosystem and then provide a privacy preserving framework for temporal changes that occur in user environment in an ad ecosystem. The online control algorithm is used to detect temporal changes in user profiling based on Lyapunov optimization to achieve an optimal solution without requiring any knowledge of future use of mobile apps or temporal changes in a user profile. We carry out extensive experimentations using mobile devices of various profiles and we examine the profiling process, the privacy leakage, privacy protection and resource usage. We develop a POC 'System App' that implements critical components of the proposed framework and further discuss its applicability in an online advertising ecosystem.

\section{REFERENCES}

[1] I. Ullah, R. Boreli, and S. S. Kanhere, "Privacy in targeted advertising: A survey," arXiv preprint arXiv:2009.06861, 2020.

[2] Y. Wang, E. Genc, and G. Peng, "Aiming the mobile targets in a cross-cultural context: Effects of trust, privacy concerns, and attitude," International Journal of Human-Computer Interaction, vol. 36, no. 3, pp. 227-238, 2020.

[3] S. Mamais, Privacy-preserving and fraud-resistant targeted advertising for mobile devices. PhD thesis, Cardiff University, 2019.
[4] I. Ullah, R. Boreli, S. S. Kanhere, S. Chawla, T. A. Ahanger, and U. Tariq, "Protecting private attributes in app based mobile user profiling," IEEE Access, vol. 8, pp. 143818-143836, 2020.

[5] Y. Liu and A. Simpson, "Privacy-preserving targeted mobile advertising: requirements, design and a prototype implementation," Software: Practice and Experience, vol. 46, no. 12, pp. 1657-1684, 2016.

[6] O. Rafieian and H. Yoganarasimhan, "Targeting and privacy in mobile advertising," Available at SSRN 3163806, 2020.

[7] G. Beigi, R. Guo, A. Nou, Y. Zhang, and H. Liu, "Protecting user privacy: An approach for untraceable web browsing history and unambiguous user profiles," in Proceedings of the Twelfth ACM International Conference on Web Search and Data Mining, pp. 213221,2019

[8] A. Razaghpanah, R. Nithyanand, N. Vallina-Rodriguez, S. Sundaresan, M. Allman, C. Kreibich, and P. Gill, "Apps, trackers, privacy, and regulators: A global study of the mobile tracking ecosystem," 2018.

[9] M. Elsabagh, R. Johnson, A. Stavrou, C. Zuo, Q. Zhao, and Z. Lin, " $\{$ FIRMSCOPE $\}$ : Automatic uncovering of privilege-escalation vulnerabilities in pre-installed apps in android firmware," in 29th \{USENIX\} Security Symposium (\{USENIX\} Security 20), 2020.

[10] J. Ren, A. Rao, M. Lindorfer, A. Legout, and D. Choffnes, "Recon: Revealing and controlling pii leaks in mobile network traffic," in Proceedings of the 14th Annual International Conference on Mobile Systems, Applications, and Services, pp. 361-374, 2016.

[11] L. Verderame, D. Caputo, A. Romdhana, and A. Merlo, "On the (un) reliability of privacy policies in android apps," arXiv preprint arXiv:2004.08559, 2020.

[12] T. Chen, I. Ullah, M. A. Kaafar, and R. Boreli, "Information leakage through mobile analytics services," in 15th International Workshop on Mobile Computing Systems and Applications, ACM HotMobile, 2014.

[13] G. A. Johnson, S. K. Shriver, and S. Du, "Consumer privacy choice in online advertising: Who opts out and at what cost to industry?," Marketing Science, 2020.

[14] I. Ullah, R. Boreli, M. A. Kaafar, and S. S. Kanhere, "Characterising user targeting for in-app mobile ads," in 2014 IEEE Conference on Computer Communications Workshops (INFOCOM WKSHPS), pp. 547-552, IEEE, 2014.

[15] I. Ullah, S. S. Kanhere, and R. Boreli, "Privacy-preserving targeted mobile advertising: A blockchain-based framework for mobile ads," arXiv preprint arXiv:2008.10479, 2020.

[16] I. Ullah, R. Boreli, S. S. Kanhere, and S. Chawla, "Profileguard: Privacy preserving obfuscation for mobile user profiles," pp. 8392, 2014.

[17] J. Parra-Arnau, D. Rebollo-Monedero, and J. Forné, "A privacypreserving architecture for the semantic web based on tag suppression," in Trust, Privacy and Security in Digital Business, pp. 5868, Springer, 2010.

[18] N. Vallina-Rodriguez, J. Shah, A. Finamore, Y. Grunenberger, K. Papagiannaki, H. Haddadi, and J. Crowcroft, "Breaking for commercials: characterizing mobile advertising," in Proceedings of the 2012 ACM conference on Internet measurement conference, pp. 343356, ACM, 2012.

[19] C. Dwork, F. McSherry, K. Nissim, and A. Smith, "Calibrating noise to sensitivity in private data analysis," in Theory of cryptography conference, pp. 265-284, Springer, 2006.

[20] C. Dwork, A. Roth, et al., "The algorithmic foundations of differential privacy.," Foundations and Trends in Theoretical Computer Science, vol. 9, no. 3-4, pp. 211-407, 2014.

[21] S. Englehardt and A. Narayanan, "Online tracking: A 1-millionsite measurement and analysis," in Proceedings of the 2016 ACM SIGSAC conference on computer and communications security, pp. 1388-1401, 2016.

[22] A. Frik, A. Haviland, and A. Acquisti, "The impact of ad-blockers on product search and purchase behavior: A lab experiment," in 29th \{USENIX\} Security Symposium ( $\{$ USENIX\} Security 20), 2020.

[23] W. Meng, R. Ding, S. P. Chung, S. Han, and W. Lee, "The price of free: Privacy leakage in personalized mobile in-apps ads.," in NDSS, 2016.

[24] A. Shuba and A. Markopoulou, "Nomoats: Towards automatic detection of mobile tracking," Proceedings on Privacy Enhancing Technologies, vol. 2, pp. 45-66, 2020.

[25] U. Iqbal, P. Snyder, S. Zhu, B. Livshits, Z. Qian, and Z. Shafiq, "Adgraph: A graph-based approach to ad and tracker blocking," in Proc. of IEEE Symposium on Security and Privacy, 2020. 
[26] G. Merzdovnik, M. Huber, D. Buhov, N. Nikiforakis, S. Neuner, M. Schmiedecker, and E. Weippl, "Block me if you can: A largescale study of tracker-blocking tools," in 2017 IEEE European Symposium on Security and Privacy (EuroSEP), pp. 319-333, IEEE, 2017.

[27] A. Das, G. Acar, N. Borisov, and A. Pradeep, "The web's sixth sense: A study of scripts accessing smartphone sensors," in Proceedings of the 2018 ACM SIGSAC Conference on Computer and Communications Security, pp. 1515-1532, 2018.

[28] X. Liu, J. Liu, S. Zhu, W. Wang, and X. Zhang, "Privacy risk analysis and mitigation of analytics libraries in the android ecosystem," IEEE Transactions on Mobile Computing, 2019.

[29] V. F. Taylor, A. R. Beresford, and I. Martinovic, "Intra-library collusion: A potential privacy nightmare on smartphones," arXiv preprint arXiv:1708.03520, 2017.

[30] M. C. Grace, W. Zhou, X. Jiang, and A.-R. Sadeghi, "Unsafe exposure analysis of mobile in-app advertisements," in Proceedings of the fifth ACM conference on Security and Privacy in Wireless and Mobile Networks, pp. 101-112, 2012.

[31] T. Book and D. S. Wallach, "A case of collusion: A study of the interface between ad libraries and their apps," in Proceedings of the Third ACM workshop on Security and privacy in smartphones E mobile devices, pp. 79-86, 2013.

[32] S. Demetriou, W. Merrill, W. Yang, A. Zhang, and C. A. Gunter "Free for all! assessing user data exposure to advertising libraries on android.," in NDSS, 2016.

[33] T. Book and D. S. Wallach, "An empirical study of mobile ad targeting," arXiv preprint arXiv:1502.06577, 2015.

[34] S. Nath, "Madscope: Characterizing mobile in-app targeted ads," in Proceedings of the 13th Annual International Conference on Mobile Systems, Applications, and Services, pp. 59-73, 2015.

[35] R. Binns, U. Lyngs, M. Van Kleek, J. Zhao, T. Libert, and N. Shadbolt, "Third party tracking in the mobile ecosystem," in Proceedings of the 10th ACM Conference on Web Science, pp. 23-31, 2018.

[36] A. Lerner, A. K. Simpson, T. Kohno, and F. Roesner, "Internet jones and the raiders of the lost trackers: An archaeological study of web tracking from 1996 to 2016," in 25th \{USENIX\} Security Symposium ( $\{$ USENIX\} Security 16), 2016.

[37] R. Binns, J. Zhao, M. V. Kleek, and N. Shadbolt, "Measuring thirdparty tracker power across web and mobile," ACM Transactions on Internet Technology (TOIT), vol. 18, no. 4, pp. 1-22, 2018.

[38] X. Pan, Y. Cao, and Y. Chen, "I do not know what you visited last summer: Protecting users from third-party web tracking with trackingfree browser," in Proceedings of the 2015 Annual Network and Distributed System Security Symposium (NDSS), San Diego, CA, 2015.

[39] F. Roesner, T. Kohno, and D. Wetherall, "Detecting and defending against third-party tracking on the web," in Proceedings of the 9th USENIX conference on Networked Systems Design and Implementation, pp. 12-12, USENIX Association, 2012.

[40] I. Ullah, B. G. Sarwar, R. Boreli, S. S. Kanhere, S. Katzenbeisser, and M. Hollick, "Enabling privacy preserving mobile advertising via private information retrieval," in 2017 IEEE 42nd Conference on Local Computer Networks (LCN), pp. 347-355, IEEE, 2017.

[41] H. Haddadi, P. Hui, and I. Brown, "Mobiad: private and scalable mobile advertising," in Proceedings of the fifth ACM international workshop on Mobility in the evolving internet architecture, pp. 33-38, ACM, 2010.

[42] M. Backes, A. Kate, M. Maffei, and K. Pecina, "Obliviad: Provably secure and practical online behavioral advertising," pp. 257-271, 2012.

[43] M. Hardt and S. Nath, "Privacy-aware personalization for mobile advertising," 2012.

[44] V. Toubiana, A. Narayanan, D. Boneh, H. Nissenbaum, and S. Barocas, "Adnostic: Privacy preserving targeted advertising," in Proceedings Network and Distributed System Symposium, 2010.

[45] S. Guha, B. Cheng, and P. Francis, "Privad: Practical privacy in online advertising," in USENIX conference on Networked systems design and implementation, pp. 169-182, 2011.

[46] M. Fredrikson and B. Livshits, "Repriv: Re-imagining content personalization and in-browser privacy," in Security and Privacy (SP), 2011 IEEE Symposium on, pp. 131-146, IEEE, 2011.

[47] R. Chen, I. E. Akkus, and P. Francis, "Splitx: High-performance private analytics," SIGCOMM Comput. Commun. Rev., vol. 43, pp. 315-326, Aug. 2013.

[48] Y. Gu, X. Gui, P. Xu, R. Gui, Y. Zhao, and W. Liu, "A secure and targeted mobile coupon delivery scheme using blockchain," in
International Conference on Algorithms and Architectures for Parallel Processing, pp. 538-548, Springer, 2018.

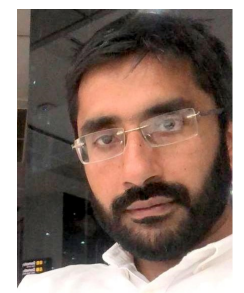

Imdad Ullah has received his Ph.D. in Computer Science and Engineering from The University of New South Wales (UNSW) Sydney, Australia. $\mathrm{He}$ is currently an assistant professor with the College of Computer Engineering and Sciences, PSAU, Saudi Arabia. He has served in various positions of Researcher at UNSW, Research scholar at National ICT Australia (NICTA)/Data61 CSIRO Australia, NUST Islamabad Pakistan and SEEMOO TU Darmstadt Germany, and Research Collaborator at SLAC National Accelerator Laboratory Stanford University USA. He has research and development experience in privacy preserving systems including private advertising and crypto-based billing systems. His primary research interest include privacy enhancing technologies; he also has interest in Internet of Things, Blockchain, network modeling and design, network measurements, and trusted networking. He regularly reviews IEEE transactions works and is also a PC Member of Cybersecurity and Privacy track of IEEE/ACM CCGRID2021. 